\title{
CD147 regulates cancer migration via direct interaction with Annexin A2 and DOCK3- $\beta$-catenin-WAVE2 signaling
}

\author{
Hong-Yong Cui ${ }^{1, *}$, Shi-Jie Wang ${ }^{1, *}$, Ji-Yu Miao ${ }^{1, *}$, Zhi-Guang Fu ${ }^{1, *}$, Fei Feng ${ }^{1}$, Jiao \\ $\mathbf{W u}^{1}{ }^{1}$, Xiang-Min Yang ${ }^{1}$, Zhi-Nan Chen ${ }^{1}$ and Jian-Li Jiang ${ }^{1}$ \\ ${ }^{1}$ Cell Engineering Research Center and Department of Cell Biology, State Key Laboratory of Cancer Biology, National Key \\ Discipline of Cell Biology, Fourth Military Medical University, Xi'an, P.R. China \\ * These authors contributed equally to the work \\ Correspondence to: Jian-Li Jiang, email: jiangjl@fmmu.edu.cn \\ Zhi-Nan Chen, email: zhinanchen@fmmu.edu.cn \\ Keywords: CD147, Annexin A2, DOCK3, WAVE2, cell movement
}

Received: June 24, $2015 \quad$ Accepted: December 12, $2015 \quad$ Published: December 22, 2015

\section{ABSTRACT}

The acquisition of inappropriate migratory feature is crucial for tumor metastasis. It has been suggested that CD147 and Annexin A2 are involved in regulating tumor cell movement, while the regulatory mechanisms are far from clear. In this study, we demonstrated that CD147 physically interacted with the $\mathrm{N}$-terminal domain of Annexin A2 and decreased Annexin A2 phosphorylation on tyrosine 23. In vitro kinase assay showed that the I domain of CD147 was indispensable for CD147-mediated downregulation of Annexin A2 phosphorylation by Src. Furthermore, we determined that p-Annexin A2 promoted the expression of dedicator of cytokinesis 3 (DOCK3) and DOCK3 blocked $\beta$-catenin nuclear translocation, resulting in inhibition of $\beta$-catenin signaling. In addition, DOCK3 inhibited lamellipodium dynamics and tumor cell movement. Also, we found that $\beta$-catenin signaling increased WAVE2 expression. Therefore, DOCK 3 was characterized as a negative regulator of WAVE2 expression via inhibiting $\beta$-catenin signaling. Our study provides the first evidence that CD147 promotes tumor cell movement and metastasis via direct interaction with Annexin $A 2$ and DOCK3- $\beta$-catenin-WAVE2 signaling axis.

\section{INTRODUCTION}

The majority of deaths associated with cancer are due to the metastasis of the original tumor cells [1]. Metastasis is an exceedingly complex and multi-step process. The acquisition of inappropriate migratory and invasive characteristics is a common feature of metastatic cancer cells. Rho family GTPases are intracellular signaling molecules that play critical roles in regulating cytoskeleton reorganization and cell movement [2]. The activities of most Rho family members depend on a delicate balance between the GTP-bound, active state and the GDP-bound, inactive state. The cycling between these two states is positively controlled by guanine nucleotide exchange factors (GEFs).

CD147 is a type I transmembrane glycoprotein. The extracellular portion of CD147 consists of an N-terminal $\mathrm{IgC} 2$ domain and a C-terminal IgI domain [3]. Previously, we reported that $\mathrm{CD} 147$ coprecipitates and colocalizes with Annexin A2 in hepatocellular carcinoma (HCC) cells and they may form a functional complex [4]. We also found that depletion of CD147 resulted in a rounded morphology, whereas the depletion of Annexin A2 produced an elongated morphology. CD147 may inhibit RhoA/ROCK signaling pathway by inhibiting Annexin A2 phosphorylation [5]. However, the evidence to support the direct interaction between CD147 and Annexin A2 is still absent and the mechanisms by which CD147 inhibits Annexin A2 phosphorylation and regulates RhoA activity are far from clear.

Annexin A2 was first identified as a major substrate of $\mathrm{v}-\mathrm{Src}$ [6]. The N-terminus possesses a phosphorylation sites on tyrosine 23 which is a substrate for phosphorylation by Src [7], serine 25 which has been reported to be phosphorylated by protein kinase $\mathrm{C}$ [8] and serine 11 which can be phosphorylated by protein kinase A [9]. Annexin A2 phosphorylation on tyrosine 23 plays an essential role in regulating cofilin-dependent actin cytoskeleton dynamics [10]. Recently, it is reported that cyclic adenosine monophosphate-induced von Willebrand 
factor secretion is promoted by serine 11 phosphorylation of Annexin A2, which is dephosphorylated via calcineurinlike phosphatase [9]. However, the negative regulation of Annexin A2 tyrosine phosphorylation is not well known.

DOCK 3 , also known as modifier of cell adhesion (MOCA), is one member of the DOCK GEF family. DOCK3 was originally identified as being one of the presenilin-binding proteins [11], is expressed in neuronal tissues, and is involved in cell adhesion and axonal degeneration $[12,13]$. DOCK3 is also described as critical for axonal outgrowth stimulating both actin rearrangement and microtubule assembly pathways $[14,15]$. In parallel of its neuronal functions, DOCK 3 is also expressed in non-neuronal cells. Sanz-Moreno et al showed that suppression of DOCK3 blocks the transition between amoeboid and mesenchymal movement [16]. Ladhani et al identified DOCK 3 as a critical regulator of Rac1 activity [17]. All these results indicate that DOCK3 regulates cytoskeleton rearrangement mainly via its GEF activity, however, whether DOCK3 could regulate cell movement independent of its GEF activity and its regulation in cancer cells are mainly unknown.

Here, we demonstrated that the I domain of CD147 interacts directly with the N-terminal domain of Annexin A2, and this interaction inhibits c-Src phosphorylation of Annexin A2 on tyrosine 23. Furthermore, DOCK3 is regulated by Annexin A2 phosphorylation and negatively regulates WAVE2 expression as an inhibitor of $\beta$-catenin signaling.

\section{RESULTS}

\section{The Ig-like domains of CD147 interact directly with Annexin A2}

We first determined the cellular localization of CD147 and Annexin A2. As shown in Figure 1a, CD147 and Annexin A2 were distributed in membrane and cytoplasm in three HCC cell lines and lung cancer A549 cells. Colocalization analysis based on Pearson's correlation coefficient (PCC) showed that there was a high degree of colocalization between CD147 and Annexin A2 (Figure 1b). We previously also performed co-localization assay using ER-Tracker to indicate ER location and we found that Annexin A2 Y23F co-localized with CD147 in cytoplasm closed to ER [5]. Also, we performed a Histag pull-down assay to confirm the interaction between CD147 and Annexin A2. As shown in Figure 1c, purified His-tagged Ig-like domains of CD147 (CD147ECP-his) could capture Annexin A2 expressed in HCC cells, and vice versa. These data indicated that CD147 may interact with Annexin A2.

To test whether CD147 interacts directly with Annexin A2 in living cells, fluorescence resonance energy transfer (FRET) assay was chosen to analyze the interaction. EGFP and DsRed were tagged to CD147 and Annexin A2, respectively. The ability and efficiency of CD147 and Annexin A2 to interact with each other were evaluated by transfecting cells with the FRET pair CD147EGFP/Annexin A2-DsRed. As shown in Figure 1d, use of the CD147-EGFP/Annexin A2-DsRed FRET pair yielded FRET efficiency significantly higher than that obtained with the negative control, where the tandem expression of EGFP and DsRed was used as positive control, which demonstrated that there is a direct interaction between CD147 and Annexin A2. We then characterized the kinetic binding parameters using purified proteins and SPR and showed that the Ig-like domains of CD147 interacted directly with Annexin A2 (Figure 1e). These parameters are typical of extracellular protein interactions measured using this technique [18].

As CD147 is known to contain two Ig-like domains [19], we then truncated CD147 (Figure 1f) and examined which Ig-like domain of CD147 was responsible for the interaction with Annexin A2. As shown in Figure 1g, both Ig-like domains of CD147 interacted with Annexin A2 as efficiently as CD147ECP. The binding parameters were also determined (Figure 1h-1i). Consistent with this, purified $\mathrm{C} 2$ domain and I domain could capture Annexin A2 expressed in HCC cells, respectively (Figure 1j).

\section{CD147 down-regulates Annexin A2 phosphorylation}

Annexin A2 is a substrate of Src and its phosphorylation by Src would induce multiple effects [10, $20,21]$. We found that Src inhibitor could downregulate Annexin A2 phosphorylation (Figure 2a), indicating that Src could phosphorylate Annexin A2 in cancer cells. Previously, we have revealed that CD147 upregulates Src activity via FAK [22], however, Tyr23 phosphorylation of Annexin A2 was negatively related to the expression of CD147 [5], which was confirmed as in Figure 2b. We found that insulin treatment could upregulate Src activity and Annexin A2 phosphorylation on Tyr23, however, elevated Annexin A2 phosphorylation induced by insulin treatment in K-7721 cells was attenuated in SMMC-7721 cells (Figure 2c). We also evaluated CD147 expression and Annexin A2 phosphorylation in HCC tissues. High CD147 expression was observed in $83.3 \%(20 / 24)$ of HCC tissues, while Annexin A2 phosphorylation on Tyr23 was below detectable level in $62.5 \%(15 / 24)$ of HCC tissues (Figure 2d). Pearson's correlation coefficient calculated between staining intensity for $\mathrm{p}-\mathrm{AnxA} 2$ and CD147 was $-0.437(p=0.033)$. To better understand this regulation, SMMC-7721 cells were transfected with plasmids to express CD147 alone or in the presence of ectopically-expressed wild-type (WT) Src or dominant positive mutant (SrcY570F). As shown in Figure 2e and 
(a)
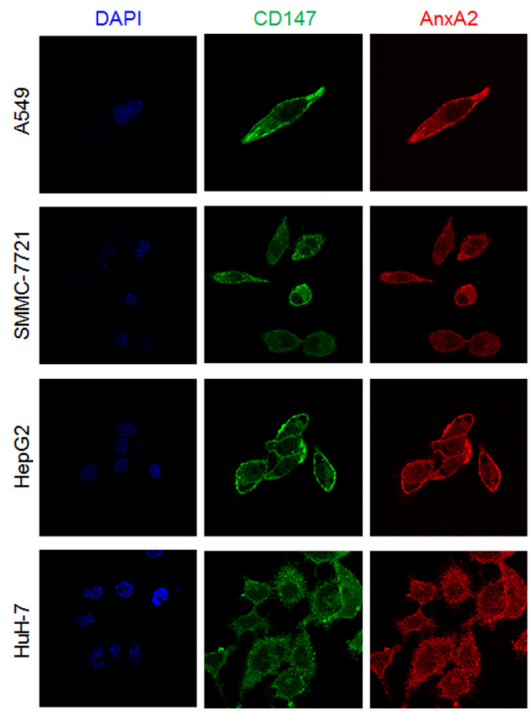

(b)

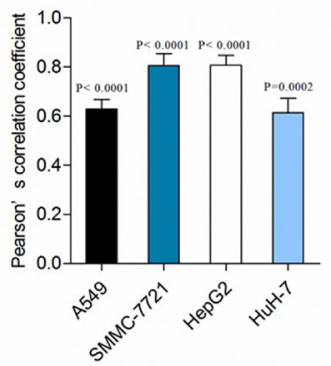

(e)

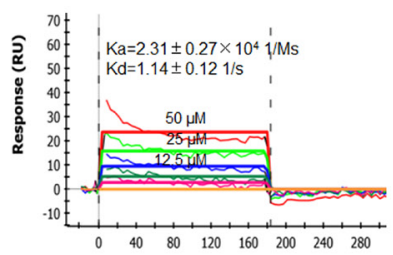

(h)

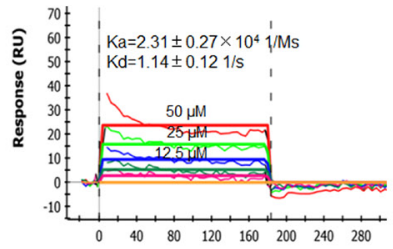

(i)

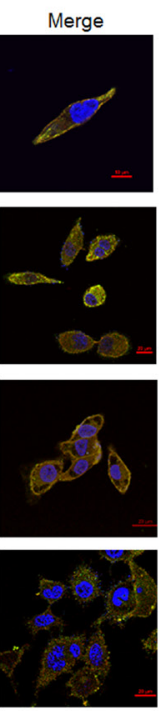

(c)
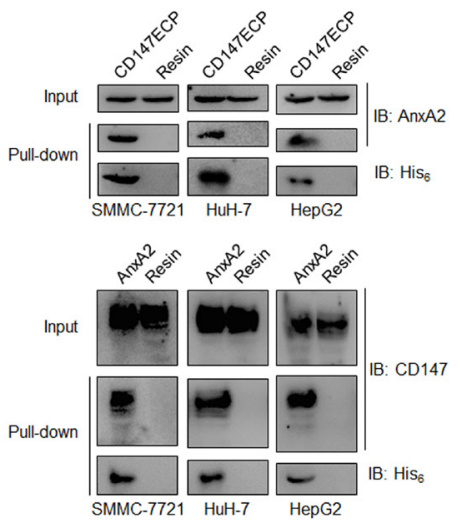

(d)

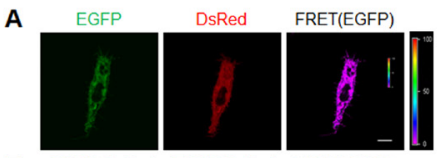

B
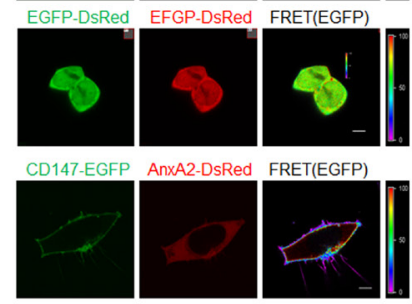

(f)

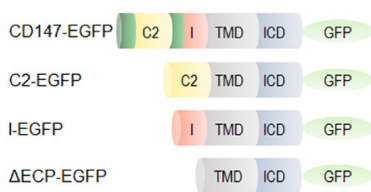

(g)

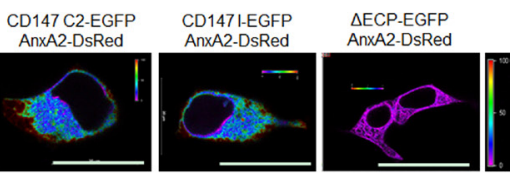

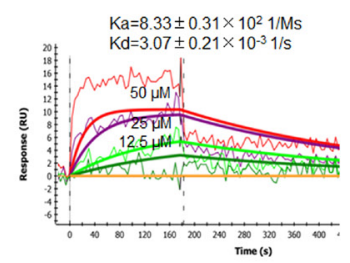

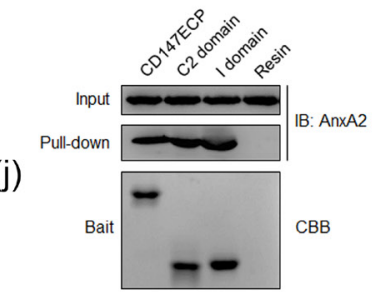

Figure 1: CD147 physically interacts with Annexin A2. a. Colocalization of CD147 and Annexin A2 in indicated cells. Scale bar, $10 \mu \mathrm{m}$ (A549 cells) or $20 \mu \mathrm{m}$ (other cells). b. Quantitative colocalization analysis of localization of CD147 and Annexin A2. The PCC values were calculated with NIS-Elements software (Nikon, Japan). The student's $t$-test was used to test the significance of PCC measurements of colocalization. c. Pull-down assay for CD147ECP-Annexin A2 interaction. d. The interaction between CD147 and Annexin A2 was analyzed

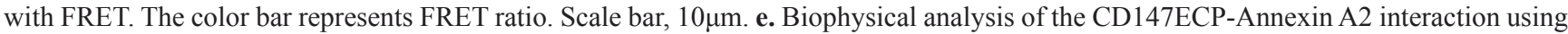
SPR. A concentration series of purified CD147ECP were injected over immobilized Annexin A2, and biophysical parameters derived from a 1:1 binding model. RU, response units. f. A schematic illustration of the structures used in FRET imaging. C2, C2 domain; I, I domain; TMD, transmembrane domain; ICD, intracellular domain. g. Interaction between the indicated constructs was analyzed with FRET. The

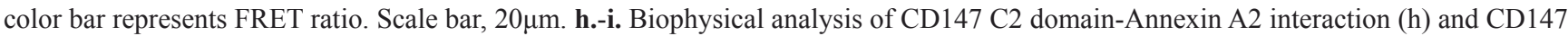
I domain-Annexin A2 interaction (i) using SPR. RU, response units. j Pull-down of endogenous Annexin A2 with purified constructs of human CD147. CBB, coomassie brilliant blue. 

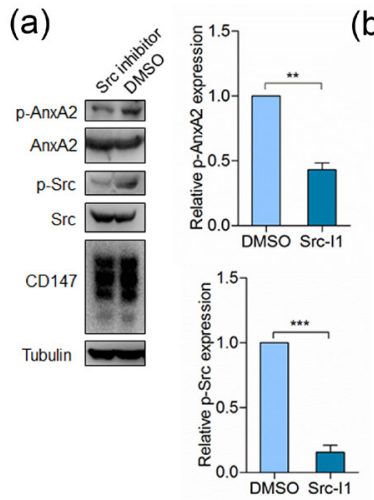

(d)
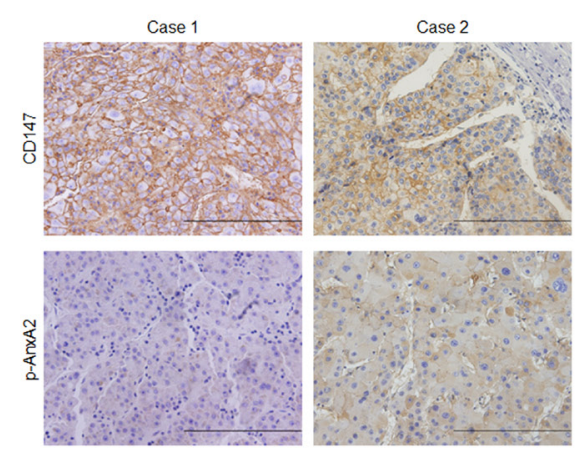

(b)

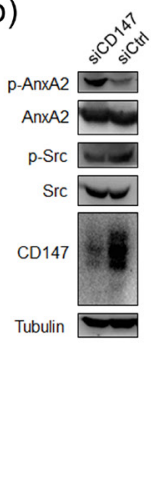

(d)

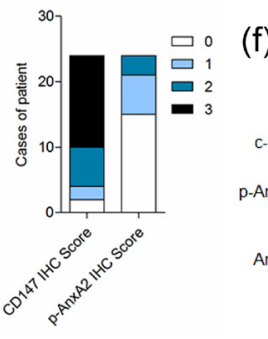

(f)
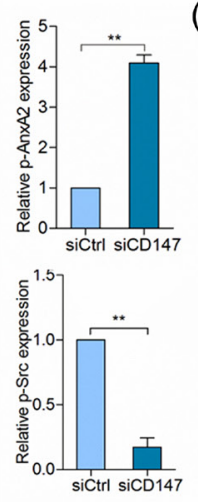

(e)

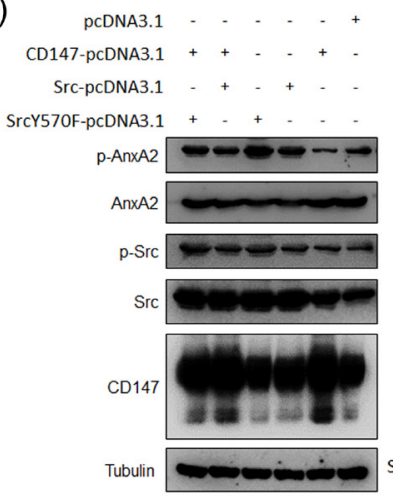

(c)

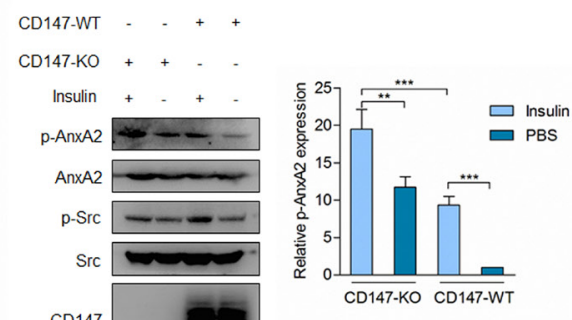

(g)
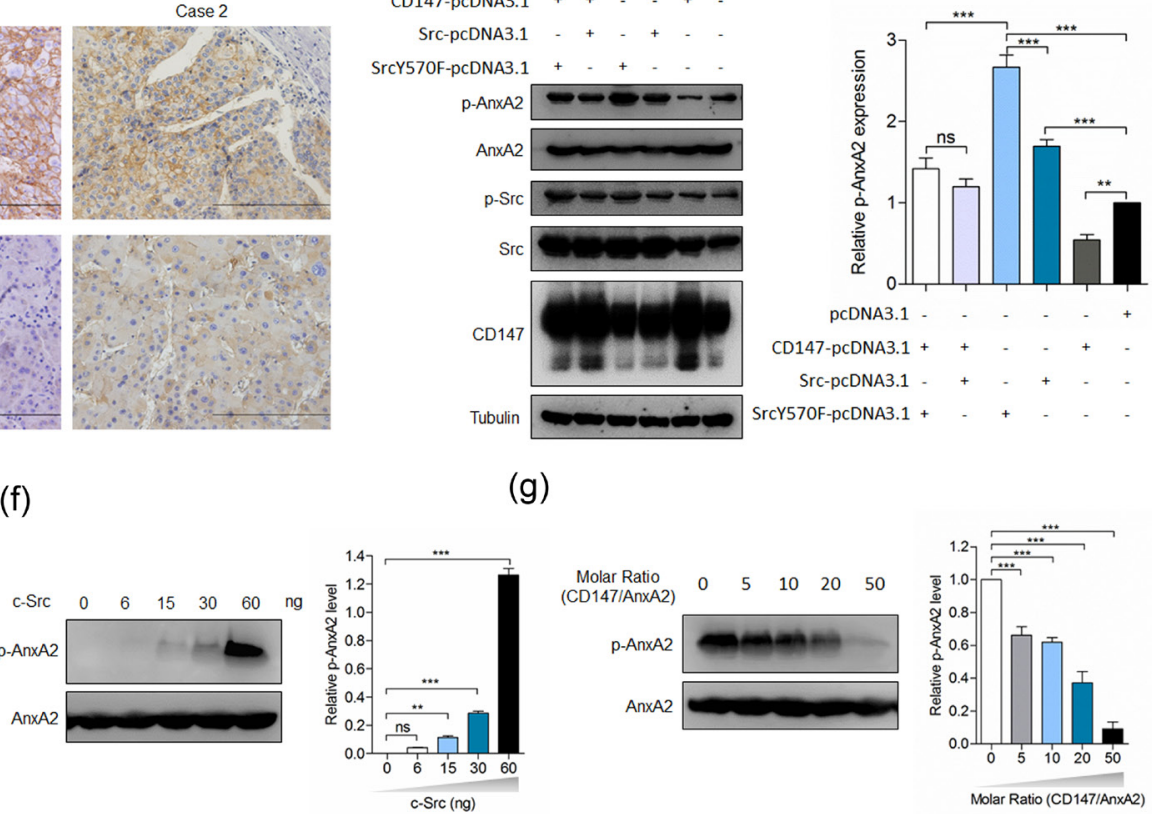

(h)

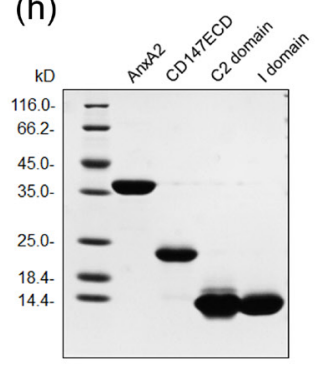

(i)

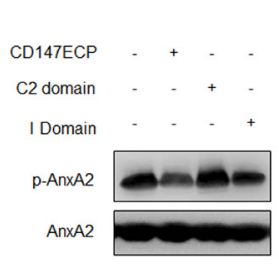

(j)

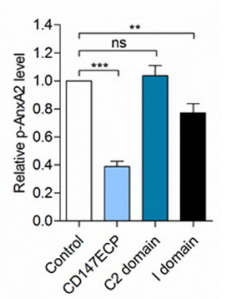

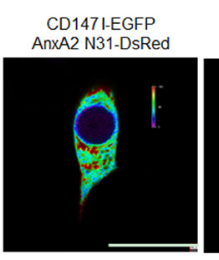

CD147 C2-EGFP AnxA2 N31-DsRed

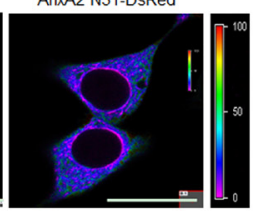

Figure 2: CD147 inhibits Annexin A2 phosphorylation by Src. a. SMMC-7721 cells were treated with Src inhibitor (Src I-1) for $24 \mathrm{~h}$ and analyzed for $\mathrm{p}$-Src and $\mathrm{p}$-Annexin A2. ${ }^{* * *} p<0.001,{ }^{* *} p<0.01$ by student's $t$-test. b. SMMC-7721 cells were transfected with a pool of siRNAs targeting CD147 (siCD147) or control siRNA (siCtrl) and analyzed for p-Src and p-Annexin A2 at $48 \mathrm{~h}$ after transfection. ${ }^{* *} p<0.01$ by student's $t$-test. c. Total cell lysates of cells in the indicated conditions were analyzed by Western blot. ${ }^{* * *} p<0.001,{ }^{* *} p<$ 0.01 by ANOVA. d. Representative images of the IHC staining. Scale bars, $200 \mu \mathrm{m}$. e. Total cell lysates of cells transfected with indicated constructs were analyzed by Western blot at $48 \mathrm{~h}$ after transfection. ${ }^{* * *} p<0.001$, ${ }^{* *} p<0.01$, ns $p>0.05$ by ANOVA. g. Src phosphorylates Annexin A2 in vitro. Indicated amounts of purified Src proteins were added to the reactions. The phosphorylation level of Annexin A2 was determined by Western blot. ${ }^{* *} p<0.001,{ }^{* *} p<0.01$, ns $p>0.05$ by ANOVA. $\mathbf{g}$. A concentration series of purified CD147ECP was added to the reactions where Src was 60ng. The phosphorylation level of Annexin A2 was determined by Western blot. $* * * p<0.001$ by ANOVA. h. Coomassie blue staining of purified proteins used in the in vitro kinase assay. i. Indicated proteins were added to the reactions and probed with phospho-specific antibody. ${ }^{* * *} p<0.001,{ }^{* *} p<0.01$, ns $p>0.05$ by ANOVA. j. Interaction between the indicated constructs was analyzed with FRET. The color bar represents FRET ratio. Scale bar, $20 \mu \mathrm{m}$. Error bars indicate the standard deviations (SD) from at least triplicate determinations $(n>3)$ 
ESM_1, Annexin A2 phosphorylation was increased in cells expressing WT Src or SrcY570F, which was attenuated in cells overexpressing CD147 together with WT Src or SrcY570F, indicating that Annexin A2 can be phosphorylated by Src and this phosphorylation can be attenuated, at least partially, by CD147.

\section{Interaction with CD147 inhibits Annexin A2 phosphorylation by Src}

To address whether CD147 interacting with Annexin A2 inhibited Annexin A2 phosphorylation by Src, an in vitro kinase assay was performed. Purified Annexin A2 was resuspended in kinase buffer, and incubated in the presence of ATP and Src. As expected, the phosphorylation level of Annexin A2 was increased with the increase of Src in the reaction system when the reaction was blotted with anti-p-Annexin A2 antibody (Figure 2f). However, when purified CD147ECP was added to the reaction system, we found that the phosphorylation level of Annexin A2 was dramatically decreased (Figure 2g), indicating that CD147 could inhibit Annexin A2 phosphorylation by Src in vitro.

As we have revealed that both Ig-like domains of CD147 could interact with Annexin A2, we supposed that both domains also could inhibit Annexin A2 phosphorylation when added into the reaction system. We purified I domain and $\mathrm{C} 2$ domain and the purity was determined by coomassie blue staining (Figure 2h). Strikingly, we found that I domain, but not C2 domain, was able to at least partially inhibit Annexin A2 phosphorylation (Figure 2i). Given that the tyrosine phosphorylation was occurred at the $\mathrm{N}$-terminal domain of Annexin A2, we determined the interaction between I domain of CD147 and the N-terminal domain of Annexin A2. We found that I domain but not C2 domain could interact directly with the N-terminal domain of Annexin A2 in living cells (Figure $2 \mathrm{j}$ ). Considering that I domain of CD147 interacts with Annexin A2 and exerts inhibitory effects on Annexin A2 phosphorylation and CD147 interacts with phosphorylation-inactive mutant of Annexin A2 but not with phospho-mimicking mutant of Annexin A2 [5], it may be a plausible explanation that this direct interaction inhibits Annexin A2 phosphorylation by Src. Taken together, these results demonstrate that I domain is necessary for CD147-mediated downregulation of Annexin A2 phosphorylation.

\section{Annexin A2 phosphorylation regulates DOCK3 expression}

Annexin A2 phosphorylation could regulate Rhomediated actin rearrangement and cell adhesion [2325]. Consistent with previous studies [26-28], we found that silencing Annexin A2 significantly enhanced cell movement (Figure 3a). Silencing CD147 inhibited cell movement, while silencing Annexin A2 simultaneously could reverse the inhibitory effect (Figure 3a), indicating that CD147 may regulate cell movement through Annexin A2. To determine the molecular mechanisms underlying cell movement regulation by Annexin A2, we transfected SMMC-7721 cells with a pool of siRNAs targeting Annexin A2, and detected the mRNA level of DOCK family GEFs. Consistent with our previous study [29], we found that silencing Annexin A2 led to DOCK3 downregulation and DOCK8 upregulation (Figure 3b). The protein expression was also verified by Western blot (Figure 3c). These results indicated that DOCK3 as a GEF may be involved in cell movement regulation by Annexin A2. Next we established an Annexin A2 stable knockdown cell line HuH-7 shA2 using lentivirus. HuH-7 shA2 cells were transfected with RNA interference-resistant plasmids to express wide-type Annexin A2 (WT), phosphorylation inactive mutant (Y23F), or phosphorylation mimic mutant (Y23E). As shown in Figure 3d, the expression of Annexin $\mathrm{A} 2$ in WT and $\mathrm{Y} 23 \mathrm{~F}$ cells was restored to that of the parent cells and the expression of Annexin A2 in Y23E cells was partially restored. However, the expression of DOCK3 in Y23E cells was fully restored and the phosphorylation inactive mutant Y23F failed to restore DOCK3 expression (Figure 3d). The protein expression level was also verified by Western blot (Figure 3e). These results demonstrated that Annexin A2 promoting DOCK3 expression is tyrosine phosphorylation dependent.

\section{DOCK3 is required for Annexin phosphorylation to regulate cell movement} A2

To investigate whether Annexin A2 phosphorylation regulates cell movement via DOCK3, we first evaluated the effects of DOCK 3 knockdown on cell motility. Figure $3 \mathrm{f}$ showed that silencing DOCK3 led to increased cell movement, similar to that of silencing Annexin A2. We then examined whether the increased DOCK3 contributed to the reduced cell motility in Annexin A2 overexpressing cells. This hypothesis predicted that reducing DOCK3 should decrease the impact of Annexin A2 overexpression on cell motility. Accordingly, we transfected $\mathrm{HuH}-7$ cells with Annexin A2-pcDNA3.1, and then selectively depleted DOCK3 by a pool of three siRNAs (Figure $3 \mathrm{~g}$ ). Cell motility was reduced in cells transfected with Annexin A2-pcDNA3.1 together with control siRNAs. Strikingly, however, Annexin A2 overexpression did not reduce cell motility to the same level in DOCK3 knockdown cells as it did in control cells. This implies that DOCK3 contributes to the regulation of cell movement by Annexin A2 phosphorylation. 
(a)
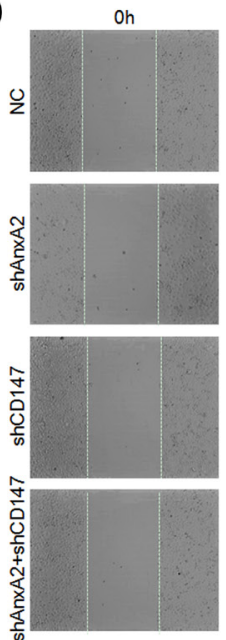

(c)

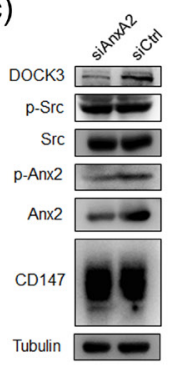

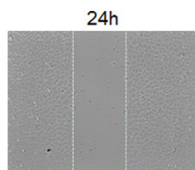
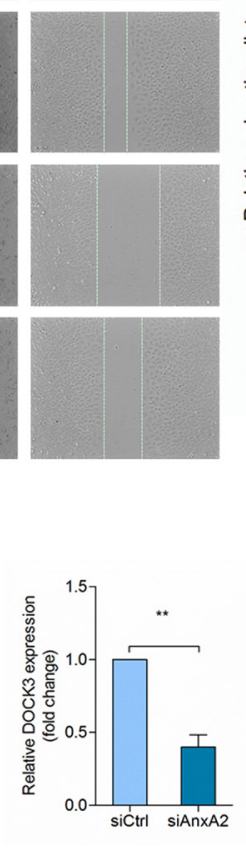

(e)

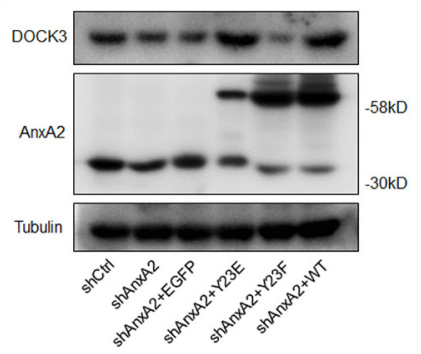

(f)

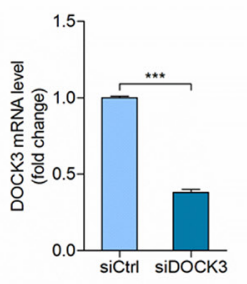

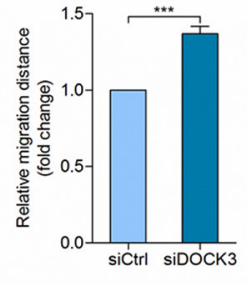

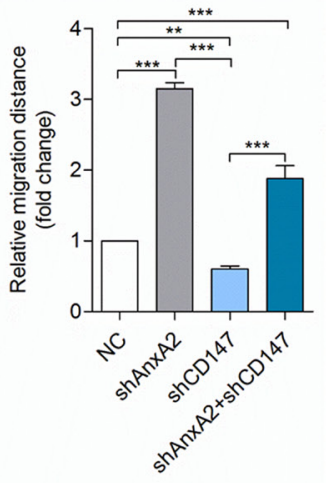

(d)

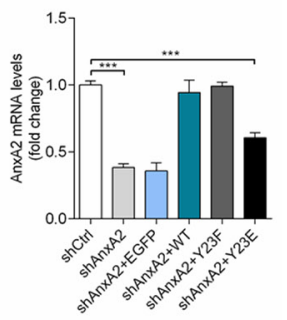

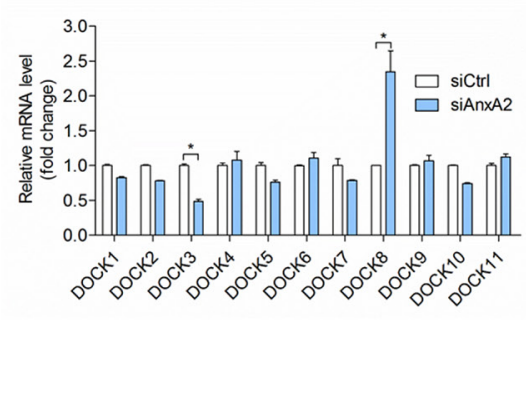

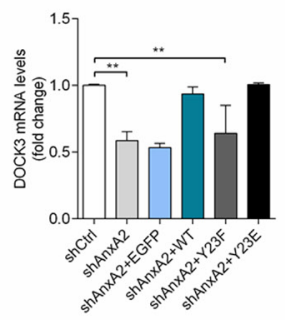

(b)

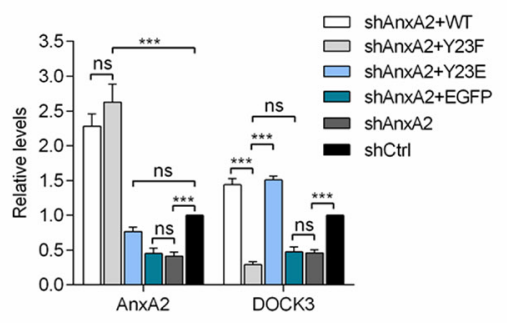

(g)
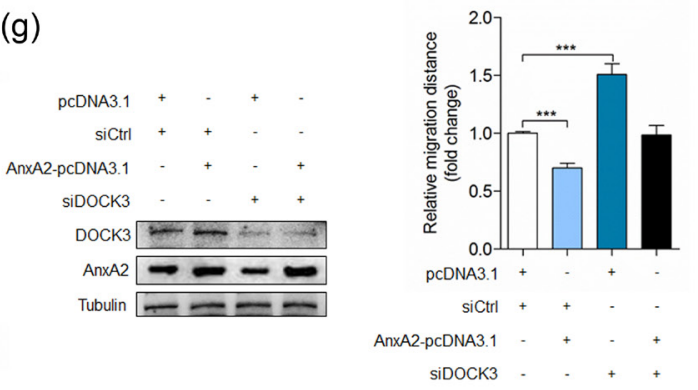

Figure 3: Annexin A2 phosphorylation regulates cell movement via DOCK3. a. Quantitative analysis of relative migration distance. $* * * p<0.001, * * p<0.01$ by ANOVA. b. Relative quantitative real-time RT-PCR analysis of DOCK family GEFs in HuH-7 cells transfected with a pool of siRNAs targeting Annexin A2 (siAnxA2) or control siRNA (siCtrl) at $24 \mathrm{~h}$ after transfection, after normalization to the expression level with siCtrl transfection. ${ }^{*} p<0.05$ by student's $t$-test. c. HuH-7 cells were transfected with siAnxA2 or siCtrl and analyzed for DOCK3 by Western blot at $48 \mathrm{~h}$ after transfection. ${ }^{* *} p<0.01$ by student's $t$-test. d. Relative quantitative real-time RTPCR analysis of Annexin A2 (left pannel) and DOCK3 (right panel) in HuH-7 cells transfected with indicated constructs at $24 \mathrm{~h}$ after transfection, after normalization to the expression level with scramble shRNA transfection. ${ }^{* * *} p<0.001,{ }^{* *} p<0.01$ by ANOVA. e. HuH-7 cells transfected with indicated constructs were analyzed for Annexin A2 and DOCK3 by Western blot at $48 \mathrm{~h}$ after transfection. $* * * p<$ 0.001 , ns $p>0.05$ by ANOVA. f. HuH-7 cells transfected with a pool of siRNAs targeting DOCK3 (siDOCK3) or siCtrl were analyzed for DOCK3 by relative quantitative real-time RT-PCR (left panel) and relative migration distance by wound-healing assay (right panel) at 24 $\mathrm{h}$ after transfection. ${ }^{* * *} p<0.001$ by student's $t$-test. g. HuH-7 cells transfected with indicated constructs were analyzed for DOCK3 and Annexin A2 by Western blot (left panel) at $48 \mathrm{~h}$ after transfection and relative migration distance by wound-healing assay (right panel) at 24 $\mathrm{h}$ after transfection. ${ }^{* * *} p<0.001$ by ANOVA. Error bars indicate the standard deviations (SD) from at least triplicate determinations $(n>3)$ 


\section{Silencing of DOCK3 increases cell motility by promoting WAVE2 expression}

DOCK 3 has been previously characterized as a GEF for Rac1 [30], therefore, we investigated whether DOCK3 regulates cell movement by influencing Rac1 activity. Silencing DOCK3 reduced Rac1 activity, which had no effect on RhoA activity (Figure 4a). Also DOCK3 could immunoprecipitate with Rac1 and negligible amount of RhoA (Figure 4b). Also, we detected the effector molecules downstream of Rac1 and RhoA signaling pathways. DOCK3 knockdown increased phosphorylation level of mlc2 (Figure 4c), however, DOCK3 knockdown strikingly increased WAVE2 expression at the mRNA and protein levels (Figure 4d). We recapitulated this effect in A549 cells (Figure 4e). Immunofluorescence staining showed that silencing DOCK3 led to increased mlc2 phosphorylation and WAVE2 expression (Figure 4f$4 \mathrm{~g})$. These results indicated that DOCK3-mediated cell movement regulation is not mainly mediated by Rac1 signaling, thus independent of its guanine nucleotide exchange activity. Furthermore, we evaluated the effects of DOCK3 knockdown on cytoskeleton rearrangement. As shown in Figure 4h, silencing of DOCK3 increased lamellipodium formation. Live-cell imaging also showed that silencing of DOCK3 increased lamellipodium formation (ESM 2-3 and Figure 4i). We also found that silencing WAVE2 led to decreased cell motility (Figure $4 \mathrm{j})$. Thus, increased cell movement induced by depletion of DOCK 3 may due to increased WAVE2 expression and lamellipodium formation.

\section{DOCK3 regulates WAVE2 expression as a negative regulator of $\beta$-catenin signaling}

We next determined the mechanism by which DOCK3 regulated WAVE2. As DOCK3 has been reported to form a complex with $\beta$-catenin and inhibit $\beta$-catenin activation [31], we wondered whether DOCK3 regulated WAVE2 expression via inhibiting $\beta$-catenin signaling. We examined the effect of DOCK3 knockdown on the localization of $\beta$-catenin. Consistent with the previous results, silencing of DOCK3 resulted in an increase in nuclear levels of $\beta$-catenin but did not affect the total amount of $\beta$-catenin (Figure 5a), indicating that DOCK3 affects the subcellular distribution of $\beta$-catenin. The effect of $\beta$-catenin activation on WAVE2 expression was evaluated in HuH-7 cells. Results shown in Figure 5b demonstrated that silencing of $\beta$-catenin led to reduced WAVE2 expression. Overexpression of a dominant active $\beta$-catenin mutant resulted in an increase of WAVE2 expression (Figure 5c). All these results argue that $\beta$-catenin activation promotes WAVE2 expression and this promotion can be repressed by DOCK3. We also evaluated the effects of GSK-3 $\beta$ inhibitor TWS119 on cell motility and found that TWS119 treatment could rescue the effects of $\mathrm{Y} 23 \mathrm{E}$ overexpression or CD147 silencing on cell motility (Figure 5d).

\section{CD147 down-regulates DOCK3 expression via inhibiting Annexin A2 phosphorylation}

We have shown that CD147 inhibits Annexin A2 phosphorylation and Annexin A2 phosphorylation induces DOCK 3 expression, thus we supposed that CD147 could inhibit DOCK3 expression and promote cell movement via interacting with Annexin A2. As expected, depletion of CD147 led to DOCK3 upregulation in indicated cell lines (Figure 6a-6b), indicating that CD147 can regulate DOCK3 expression. To further confirm the regulation mechanisms presented in this study, we also detected the other key molecules. We found that CD147 deletion resulted in increased Annexin A2 phosphorylation and decreased WAVE2 expression and Src activation (Figure $6 \mathrm{~b})$. Overexpression of phosphorylation mimic mutant Y23E dramatically attenuated the effects of CD147 overexpression on DOCK3 and WAVE2 expression (Figure 6c) and cell motility (Figure 6d). Furthermore, WAVE2 silencing rescued the effect of DOCK3 knockdown on cell motility and DOCK3 silencing rescued the effect of Y23E overexpression on cell motility (Figure 6e). Taken together, these results demonstrated that CD147 can promote cell movement via interacting with Annexin A2 and reducing DOCK 3 expression, resulting in increased WAVE2 expression.

\section{CD147 promotes HCC metastasis in nude mice}

Cytoskeletal rearrangement and cell motility achieve dire significance during tumor metastasis. We investigated whether CD147 influenced tumor metastasis in vivo. The incidence of metastasis after intra-splenic injection of SMMC-7721 or K-7721 cells was shown in Table 1. The metastasis was substantially suppressed in K-7721 group $(p<0.05)$ compared with that in SMMC-7721 group. As shown in Table 1 and Figure 7a, injection of SMMC7721 cells into spleen resulted in 6 of 8 mice developing more than 50 visible liver metastases. However, only 1 of 8 mice in K-7721 group developed more than 50 visible liver metastases. IHC staining showed CD147 suppressed DOCK3 expression and enhanced WAVE2 expression (Figure 7a).

\section{DISCUSSION}

In this study, we found that the I domain of CD147 could bind the N-terminal domain of Annexin A2 and inhibit Annexin A2 phosphorylation on tyrosine 23 by Src. Phosphorylation of Annexin A2 promoted the expression of DOCK3 and DOCK3 suppressed the expression of 
(a)

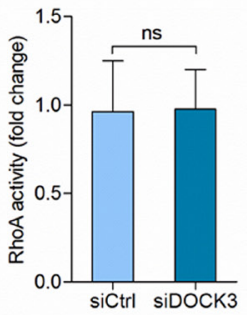

(d)

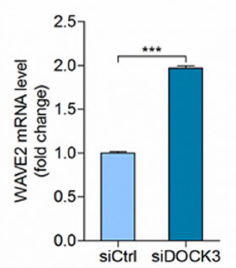

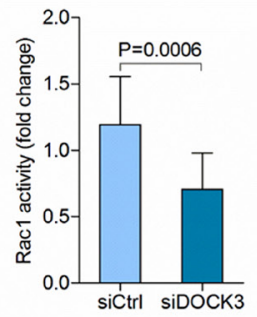

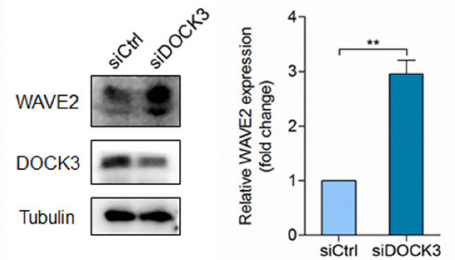

(b)

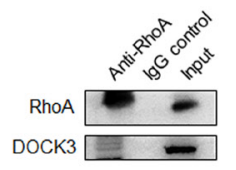

(c)

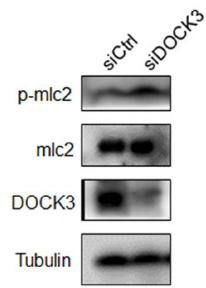

(e)
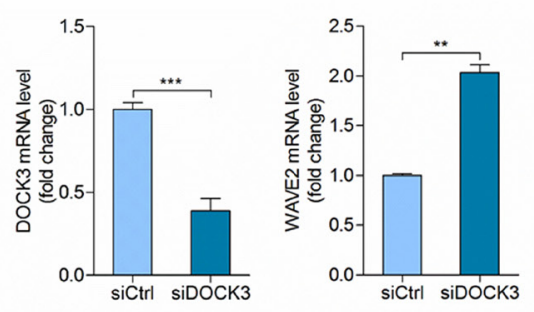

(f)
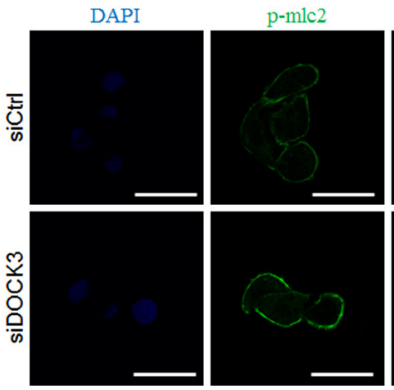

(h)

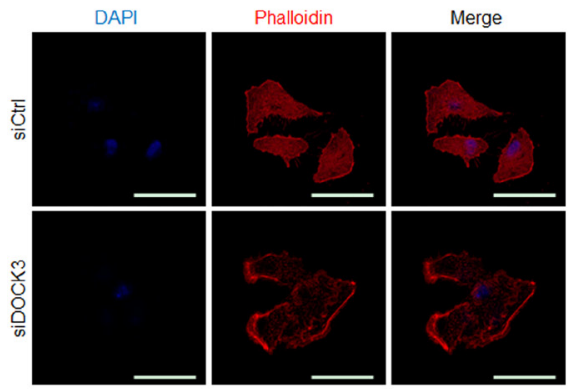

(j)
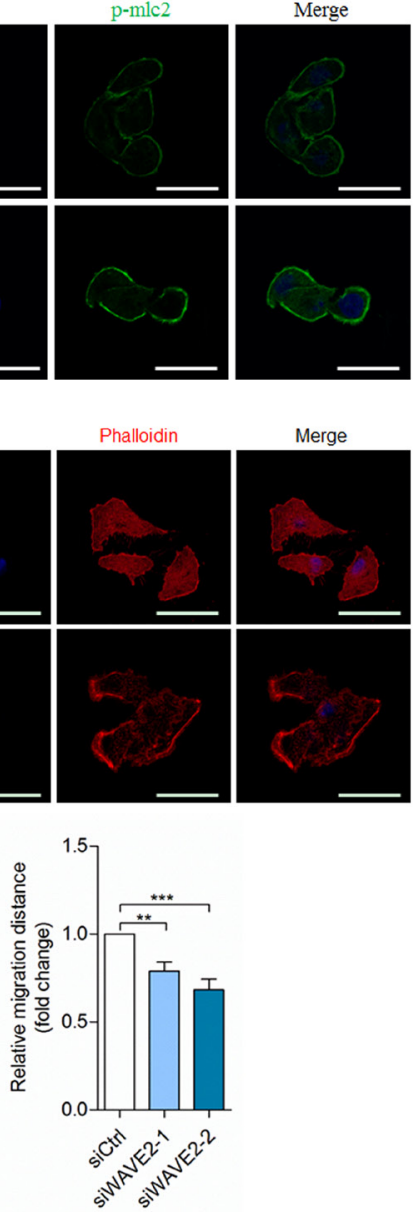

(g)

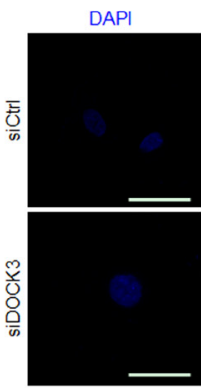

WAVE2

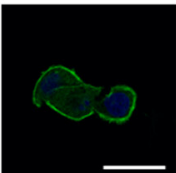

(i)

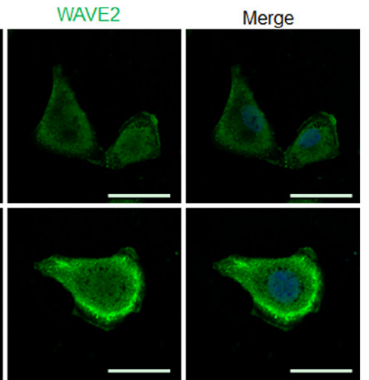

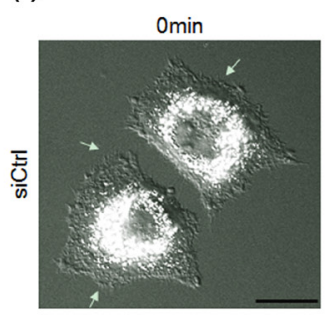

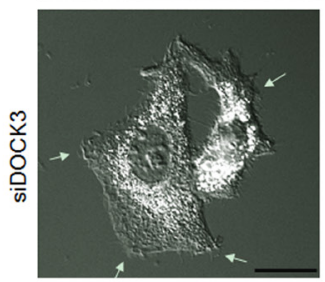

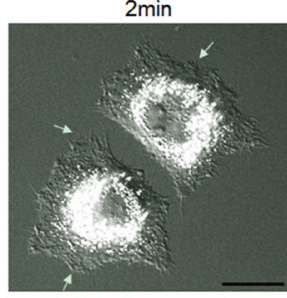

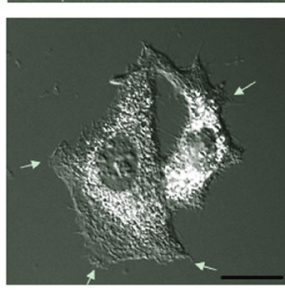

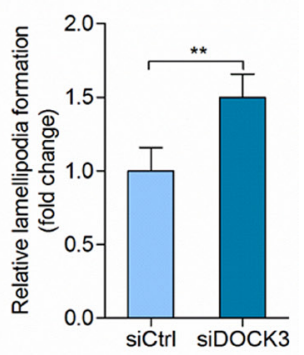

Figure 4: DOCK3 inhibits WAVE2 expression. a. Quantification of RhoA (left panel) and Rac1 (right panel) activation in HuH-7 cells transfected with siDOCK 3 or siCtrl at $48 \mathrm{~h}$ after transfection. ns $p>0.05$ by student's $t$-test. b. Co-immunoprecipitation of endogenous RhoA (left panel) or Rac1 (right panel) with DOCK3. c. HuH-7 cells transfected with siDOCK3 or siCtrl were analyzed for p-mlc2 and DOCK 3 by Western blot at $48 \mathrm{~h}$ after transfection. d. HuH-7 cells transfected with siDOCK 3 or siCtrl were analyzed for WAVE2 by relative quantitative real-time RT-PCR (left panel) and Western blot (right panel) at $48 \mathrm{~h}$ after transfection. ${ }^{* * *} p<0.001$ by student's $t$-test. e. Relative quantitative real-time RT-PCR analysis of DOCK3 (left panel) and WAVE2 (right panel) in A549 cells transfected with siDOCK3 or siCtrl at $48 \mathrm{~h}$ after transfection. ${ }^{* * *} p<0.001,{ }^{* *} p<0.01$ by student's $t$-test. f.-h. Confocal microscopy images of p-mlc2 expression (f), WAVE2 expression (g) and actin cytoskeleton rearrangement $(\mathrm{h})$ in A549 cells transfected with siDOCK3 or siCtrl at $48 \mathrm{~h}$ after transfection. i. DIC microscopy images of A549 cells transfected with siDOCK3 or siCtrl at $48 \mathrm{~h}$ after transfection. Arrows indicate lamellipodium dynamics. ${ }^{* *} p<0.01$ by student's $t$-test. j. Quantitative analysis of relative migration distance. ${ }^{* * *} p<0.001,{ }^{* *} p<0.01$ by ANOVA. Error bars indicate the standard deviations (SD) from at least triplicate determinations $(n>3)$ 
Table 1: Number of mice with metastasis in each group

\begin{tabular}{|l|l|l|}
\hline No. Metastasis in Each Mouse & CD147-WT, $\boldsymbol{n}(\%)$ & CD147-KO, $\boldsymbol{n}(\%)^{\#}$ \\
\hline$<50$ & $2(25 \%)$ & $7(87.5 \%)$ \\
\hline$>50$ & $6(75 \%)$ & $1(12.5 \%)$ \\
\hline Total & 8 & 8 \\
\hline
\end{tabular}

${ }^{\#}$ CD147-KO versus CD147-WT: $p<0.05$

WAVE2 via inhibiting $\beta$-catenin signaling. Thus CD147 promoted cell movement via $\mathrm{p}$-Annexin A2-DOCK3- $\beta$ catenin-WAVE2 signaling axis (Figure 7b).

One of the cellular functions of Annexin A2 is its involvement in cytoskeletal rearrangement, which is thought to be mediated by its tyrosine phosphorylation and its regulation of RhoA [5, 10, 23], indicating that Annexin A2 seems to regulate actin remodeling by acting upstream of RhoA. Whether Annexin A2 regulates Rac activity is not clear. Previous studies suggest that DOCK3 functions mainly dependent on its GEF catalytic activity in regulating cytoskeletal reorganization. We identified DOCK3 as a tyrosine phosphorylated Annexin A2regulated RacGEF (Figure 4), we therefore hypothesized that p-Annexin A2 may regulate cytoskeleton rearrangement through DOCK3-mediated activation of Rac1 signaling. Consistent with previous studies, DOCK3 co-precipitates with Rac1 and silencing DOCK3 leads to reduced Rac1 activation. However, WAVE2, generally considered as a downstream effector of Rac1 signaling $[32,33]$, is dramatically increased both at mRNA and protein levels, indicating that DOCK3 may regulate WAVE2 independent of its guanine nucleotide exchange activity and Rac1 signaling. It may be plausible that the down-regulation of WAVE2 resulted from Rac1 inhibition can be greatly offset by the up-regulation of WAVE2 resulted from DOCK3 silence, thus leading to decreased Rac1 activity while increased WAVE2 expression. We also found that Annexin A2 suppressed DOCK8 expression, indicating that DOCK8 may be involved in Annexin A2- (a)

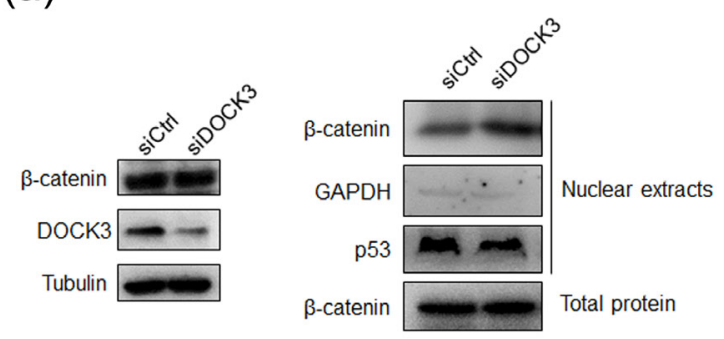

(b)
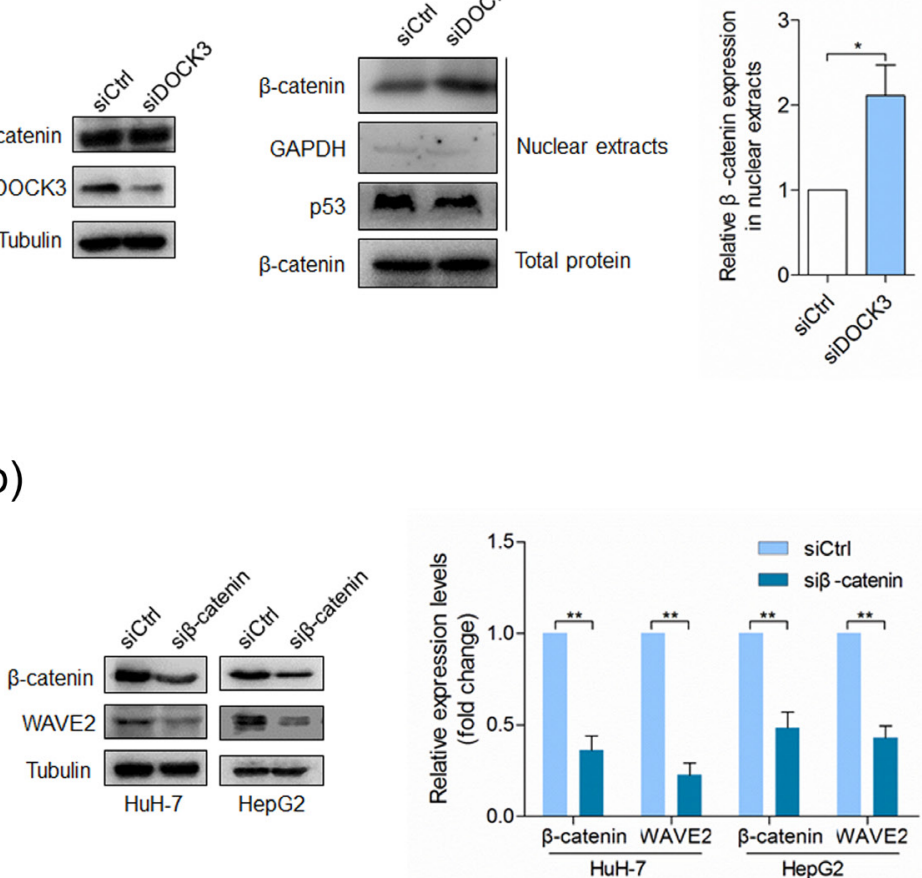

(c)
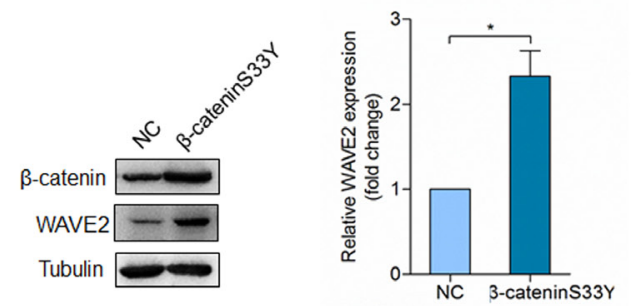

(d)

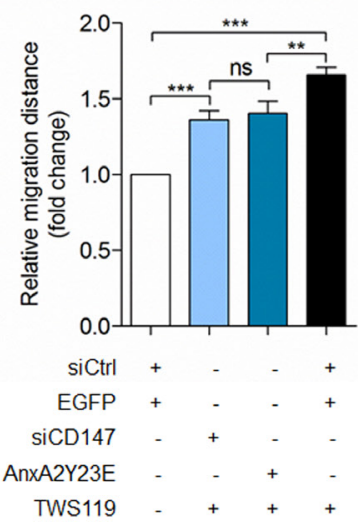

Figure 5: DOCK3 inhibits WAVE2 expression as a negative regulator of $\boldsymbol{\beta}$-catenin signaling. a. Western blot analysis for $\beta$-catenin and DOCK3 at $48 \mathrm{~h}$ after transfection with siDOCK3 or siCtrl (left panel). Western blot analysis for $\beta$-catenin, p53 and GAPDH in nuclear extracts at $48 \mathrm{~h}$ after transfection with siDOCK3 or siCtrl (middle panel). Quantitative analysis of nuclear-total ratio of $\beta$-catenin (right panel) $p<0.05$ by student's $t$-test. b. Western blot analysis for $\beta$-catenin and WAVE2 in cells transfected with a pool of siRNAs targeting $\beta$-catenin (si $\beta$-catenin) or siCtrl at $48 \mathrm{~h}$ after transfection. ${ }^{* *} p<0.01$ by student's $t$-test. c. Western blot analysis for WAVE2 in HuH-7 cells transfected with pcDNA3 or $\beta$-cateninS33Y-pcDNA3 at $48 \mathrm{~h}$ after transfection. ${ }^{*} p<0.05$ by student's $t$-test. d. Quantitative analysis of relative migration distance. ${ }^{* *} p<0.001,{ }^{* *} p<0.01$, ns $p>0.05$ by ANOVA. Error bars indicate the standard deviations (SD) from at least triplicate determinations $(n>3)$ 
(a)

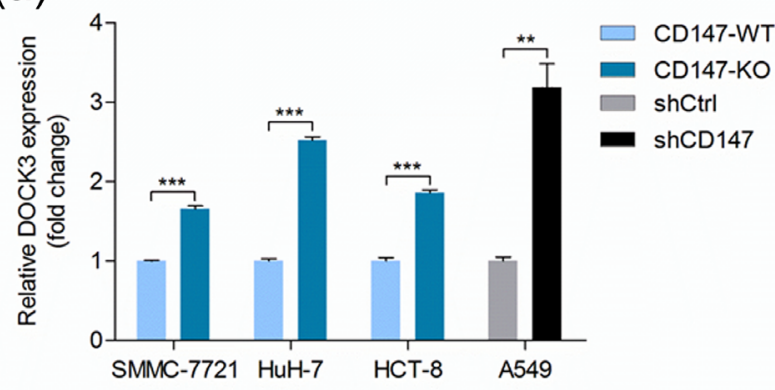

(c)
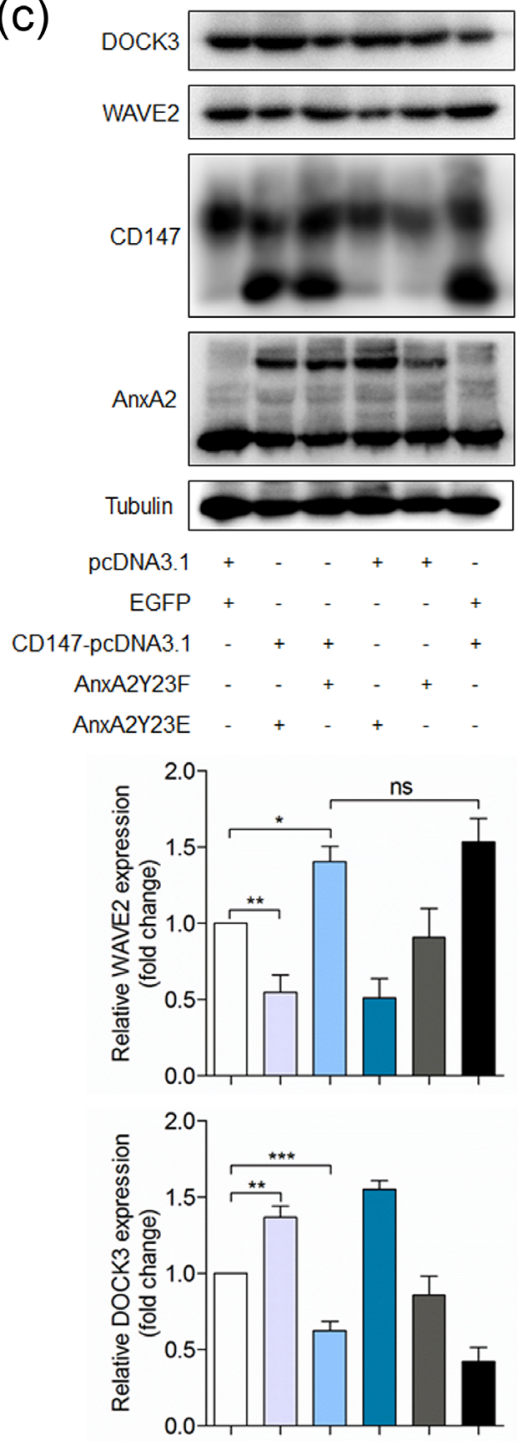

(b)

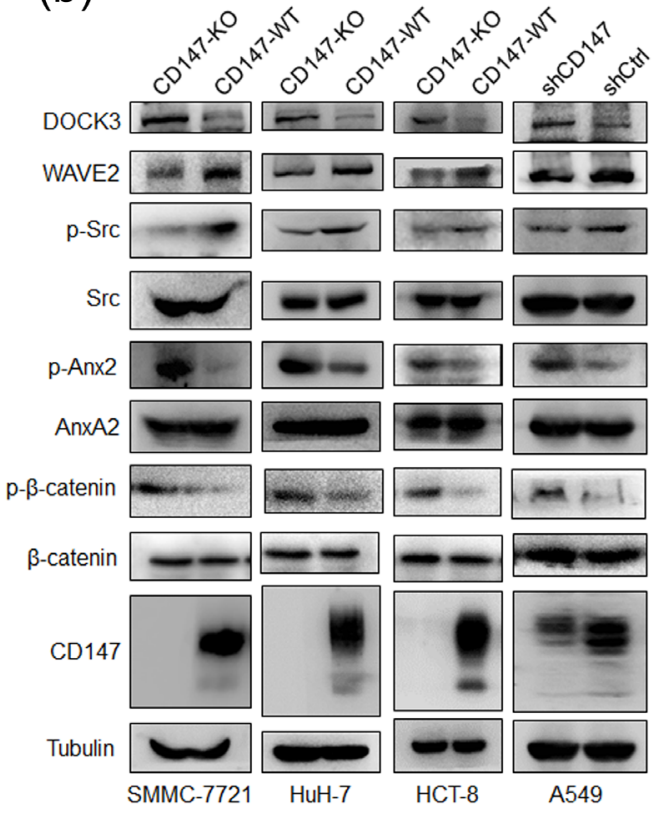

(d)

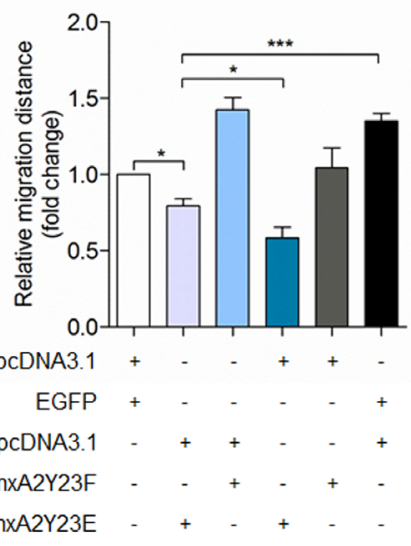

(e)

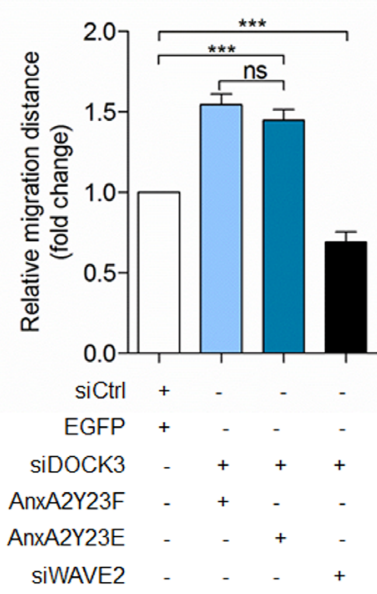

Figure 6: CD147 promotes cancer cell migration via inhibiting Annexin A2 phosphorylation and DOCK3 expression. a. Relative quantitative real-time RT-PCR analysis of DOCK3 in indicated cell lines. ${ }^{* * *} p<0.001,{ }^{* *} p<0.01$ by student's $t$-test. $\mathbf{b}$. Western blot analysis for DOCK3, WAVE2, p-Src, p- $\beta$-catenin and p-Annexin A2 in indicated cells. c. Western blot analysis for DOCK3 and WAVE2 in cells transfected with indicated constructs. ${ }^{* * *} p<0.001,{ }^{* *} p<0.01,{ }^{*} p<0.05$, ns $p>0.05$ by ANOVA. d.-e. Quantitative analysis of relative migration distance. ${ }^{* * *} p<0.001,{ }^{* *} p<0.01,{ }^{*} p<0.05$, ns $p>0.05$ by ANOVA. Error bars indicate the standard deviations (SD) from at least triplicate determinations $(n>3)$ 
(a)
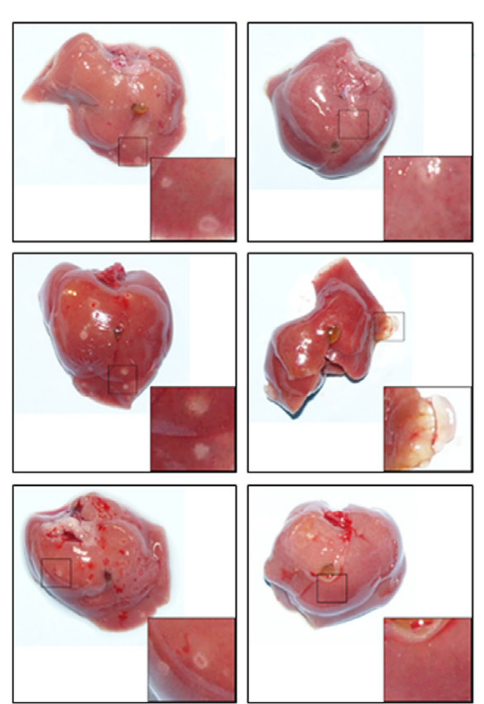

CD147-WT

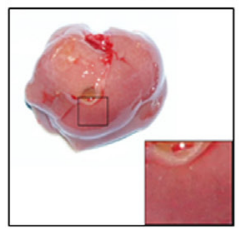

CD147-KO
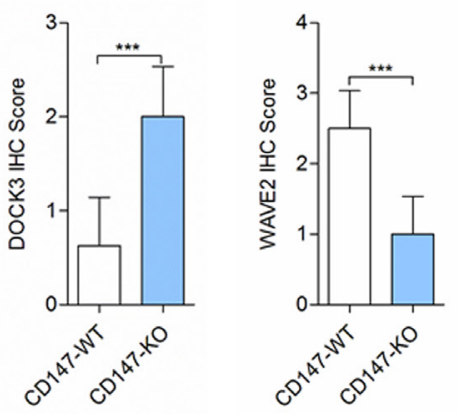
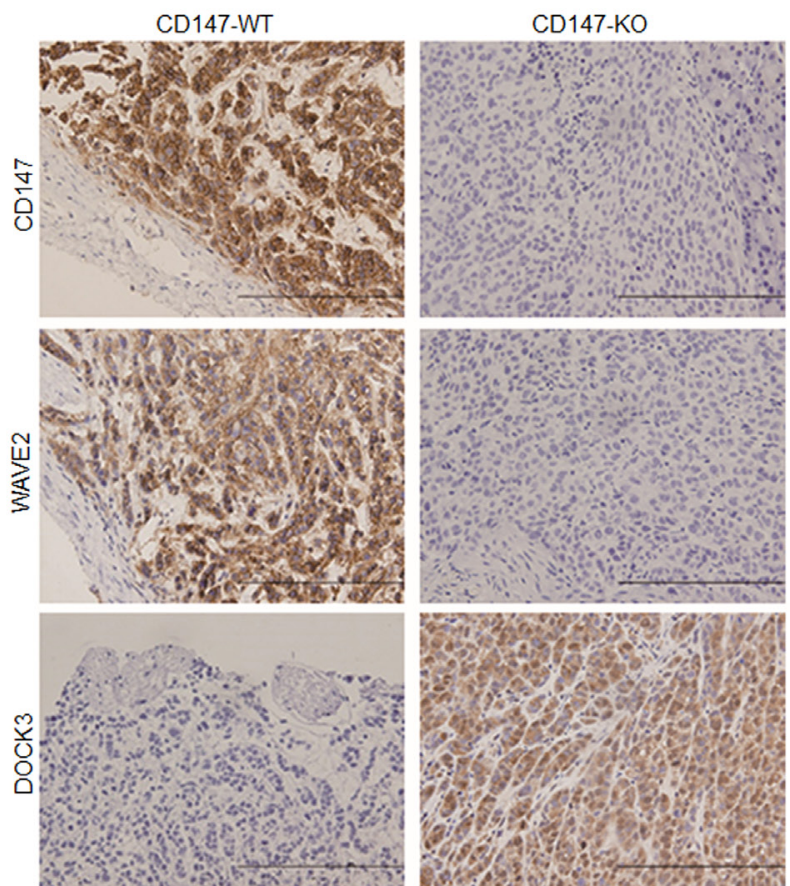

(b)

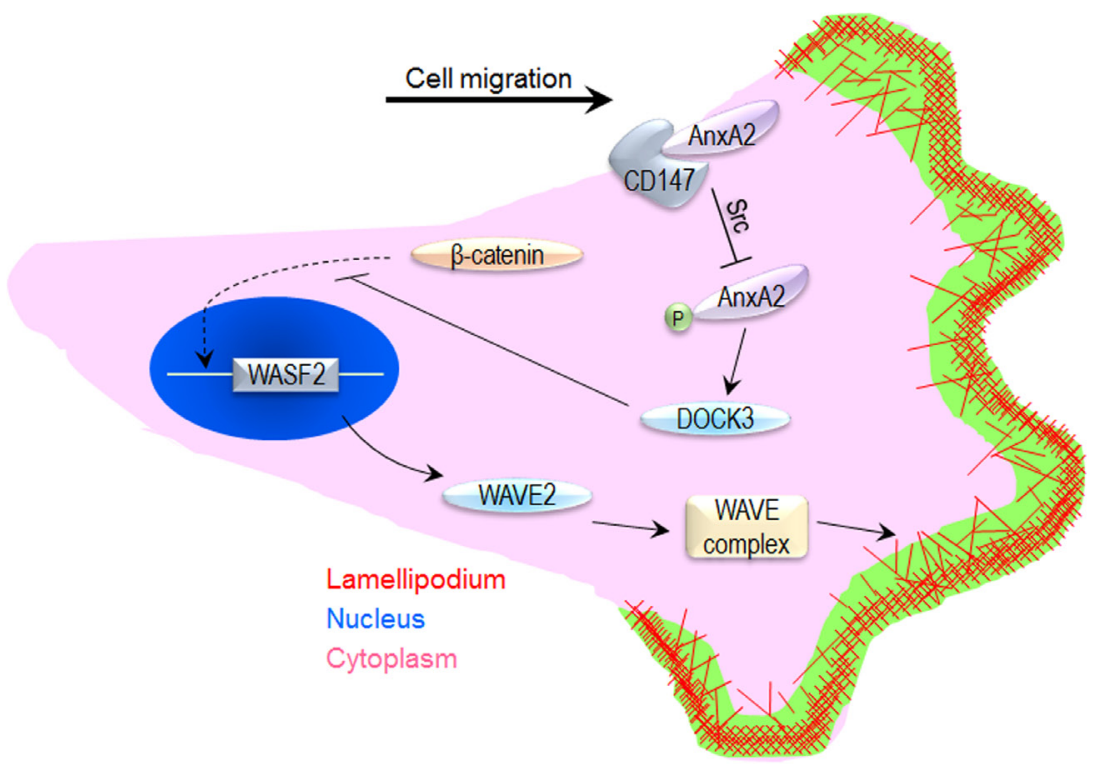

Figure 7: CD147 promotes cancer metastasis. a. Metastasis assay by intra-splenic injection of SMMC-7721 or K-7721 cells in nude mice. Livers were excised for examination (left panel). Tumors were assessed by histology (right panel). Expression of CD147, WAVE2 and DOCK3 were examined by immunohistochemistry. Scale bar, $200 \mu \mathrm{m}$. ${ }^{* * *} p<0.001$ by student's $t$-test. b. Schematic representation of the major mechanisms of CD147 in regulating cancer cell movement via interaction with Annexin A2. mlc2: myosin light chain 2; DOCK3: dedicator of cytokinesis 3; WAVE2: Wiskott-Aldrich syndrome family verprolin-homologous protein 2. 
regulated cancer migration and progression.

CD147 has been reported to play important roles in cellular processes of HCC progression, including adhesion, migration and invasion [34-36]. Of note, CD147 interacts with integrin and increases $\alpha 3 \beta 1$ and $\alpha 6 \beta 1$ activity, enhances expression and phosphorylation of FAK and paxillin, and subsequently leads to cytoskeletal rearrangement and changes of cell morphology [37-39]. Also, it is believed that CD147 plays a role in mediating epithelial-mesenchymal transition (EMT) in the process of HCC progression, providing a slight clue to the function of CD147 in cytoskeleton rearrangement [40]. Recently, we reported that $\mathrm{CD} 147$ promotes cell motility by regulating Annexin A2-activated RhoA [5], enhances Src activity and promotes mesenchymal-type cell movement by upregulating DOCK8 [22]. All these results, together with our data reported here, suggest that CD147 regulates cytoskeleton rearrangement and cell movement at multiple levels and through different mechanisms, although how to coordinate these mechanisms during regulating cell movement at spatio-temporal level still awaits further investigation and it is still difficult to dissect to what extent each mechanism contributes to regulation of cell movement under diverse circumstances. Above all, targeting CD147 could be a promising strategy to reduce migration and metastasis of tumor cells.

\section{MATERIALS AND METHODS}

\section{Antibodies and biosensors}

Antibodies specific for CD147 (sc-9754), $\alpha$-tubulin (sc-8035), WAVE2 (sc-10392), Annexin A2 (sc-48397), p-Annexin A2 (sc-135753), RhoA (sc-418) and donkey anti-goat IgG-FITC (sc-2024) were purchased from Santa Cruz (Dallas, Texas); p-Src (Tyr416) (2013), p-myosin light chain 2 (Thr18/Ser19) (3674), Src (2109), $\beta$-catenin (8814) and myosin light chain 2 (3672) antibodies were purchased from Cell Signaling Technology (Boston, MA); DOCK3 antibody (orb156627) was obtained from Biorbyt (Cambridge, UK); and Alexa 594-conjugated goat antimouse IgG and Alexa Fluor 488 phalloidin were purchased from Invitrogen (Carlsbad, CA). Rac1 antibody (ARC03) was obtained from Cytoskeleton (Denver, CO). The Src kinase inhibitor Src I-1 (Sigma, St. Louis, MO) was used at $300 \mathrm{nM}$. The GSK-3 $\beta$ inhibitor TWS119 (sc-221694, Santa Cruz, Texas) was used at $5 \mu \mathrm{M}$. The RhoA biosensor was obtained from Addgene. The Rac1 biosensor was a gift from Dr. Louis Hodgson of Yeshiva University.

\section{Tissue specimens and IHC analysis}

24 tissue specimens of HCC were collected from the Department of Hepatobiliary \& Pancreas Surgery, Xijing Hospital, which is affiliated with the Fourth Military Medical University (FMMU) from 2008 to 2009 and were histological confirmed by staining with hematoxylin and eosin (H\&E). All individuals provided written informed consent, and the study was approved by the hospital Ethics Committee. For IHC analysis, sections were incubated with primary antibodies and developed with the Histostain ${ }^{\circledR}$-Plus Kit (Invitrogen, Carlsbad, CA). The expression level was independently evaluated by 2 senior pathologists according to the proportion and intensity of positive cells. The following criteria were used to score each specimen: 0 (no staining), 1 (any percentage with weak intensity or $<30 \%$ with intermediate intensity), 2 ( $>30 \%$ with intermediate intensity or $<50 \%$ with strong intensity) or 3 ( $>50 \%$ with strong intensity).

\section{Cell lines}

Human SMMC-7721 hepatoma cell line was obtained from Chinese Academy of Medical Sciences. HuH-7 cells were obtained from the Japanese Collection of Research Bioresources. HepG2, A549 and HCT-8 cells were obtained from the American Type Culture Collection. All cell lines were routinely cultured using standard protocols. A CD147-knockout SMMC-7721 cell line (K-7721) was established using the zinc finger nuclease (ZFN)-targeting approach [41]. Cell line authentication was assessed using short tandem repeat (STR) DNA profiling method in our laboratory and the latest verification was done in March 2014.

\section{RNA interference}

Cells were transfected with a pool of siRNAs using HiPerFect Transfection Reagent according to the manufacturer's instruction (Qiagen). siRNA mixes targeting Annexin A2, CD147, DOCK3, WAVE2 or $\beta$-catenin were designed and synthesized by Shanghai GenePharma Co. (Shanghai, China). Silencer negative control siRNA (siCtrl) was used as a negative control under similar conditions. shRNA set (4 individual hairpins) against Annexin A2 and shRNA set (2 individual hairpins) against CD147 were obtained from Shanghai Genechem Co. (Shanghai, China) and used to generate lentiviral particles in packaging cells. The pre-made negative control shRNA served as negative control. 
Table 2: Primer sequences used in Quantitative Real-time PCR

\begin{tabular}{|c|c|c|}
\hline DOCK1 & Forward & 5'-ACCGAGGTTACACGTTACGAA-3' \\
\hline DOCK1 & Reverse & 5'-TCGGAGTGTCGTGGTGACTT-3' \\
\hline DOCK2 & Forward & 5'-AGCACAAAATGTTACAGGGCA-3' \\
\hline DOCK2 & Reverse & 5'-AGCACAAAATGTTACAGGGCA-3' \\
\hline DOCK3 & Forward & 5'-TATGCAGCTTTCGAGGATCTGT-3' \\
\hline DOCK3 & Reverse & 5'-GCCCATTCTTGTAGAGTTGCT-3' \\
\hline DOCK4 & Forward & 5'-GGATACCTACGGAGCACGAG-3' \\
\hline DOCK4 & Reverse & 5'-AGCCATCACACTTCTCCAGG-3' \\
\hline DOCK5 & Forward & 5'-CTGTAGCAGCCTTAGTCGCC-3' \\
\hline DOCK5 & Reverse & 5'-GCAAGGAGAGCTCCACATCT-3' \\
\hline DOCK6 & Forward & 5'-TGTATGATGTGCGGGAGAAAAA-3' \\
\hline DOCK6 & Reverse & 5'-AGGGGTAGGTCACAGAGAAGA-3' \\
\hline DOCK7 & Forward & 5'-TGGGCCTGTAGTATCTTTGACT-3' \\
\hline DOCK7 & Reverse & 5'-TCTTCCTCATCTGGTGATGGAT-3' \\
\hline DOCK8 & Forward & 5'-ACGCGCCGTGTAACTGTGAA-3' \\
\hline DOCK8 & Reverse & 5'-CCCCGAGCTCCTGGGCAA-3' \\
\hline DOCK9 & Forward & 5'-GCTTCCGAACAAAGTGGTCAA-3' \\
\hline DOCK9 & Reverse & 5'-GCCATGCTTGGTGATCCCA-3' \\
\hline DOCK10 & Forward & 5'-CCGTATGCCTTTTGCTTGGG-3' \\
\hline DOCK10 & Reverse & 5'-GCTTATTCTGTCGGCCCTTCT-3' \\
\hline DOCK11 & Forward & 5'-AGAGGCTGCGATGTGTTATGT-3' \\
\hline DOCK11 & Reverse & 5'-CTCATAAATTGGAACGATCAACT-3' \\
\hline WAVE2 & Forward & 5'-CTTTCAGCCATCCGTCAAGG-3' \\
\hline WAVE2 & Reverse & 5'-AATCGGACCAGTCGTCCTCA-3' \\
\hline Annexin A2 & Forward & 5'-GAGGATGGCTCTGTCATTGATT-3' \\
\hline Annexin A2 & Reverse & 5'-CTGGTAGTCGCCCTTAGTGTCT-3' \\
\hline CD147 & Forward & 5'-ACTCCTCACCTGCTCCTTGA-3' \\
\hline CD147 & Reverse & 5'-GCCTCCATGTTCAGGTTCTC-3' \\
\hline GAPDH & Forward & 5'-GCACCGTCAAGGCTGAGAAC-3' \\
\hline GAPDH & Reverse & 5'-TGGTGAAGACGCCAGTGGA-3' \\
\hline
\end{tabular}

\section{Generation of CD147 knockout cell lines with the CRISPR/Cas9 system}

CD147 knockout HuH-7 and HCT-8 cell lines were prepared by Beijing Biocytogen Co. Ltd. using EGE ${ }^{\mathrm{TM}}$ system. Part of CD147 coding region (from 3' part of exon 2 to exon 8) was replaced with puromycin resistant genes flanked by 5' and 3' homologous arm ( $1 \mathrm{~kb}$ each) respectively, in the donor plasmids. Cas9/sgRNA plasmid was designed against exon 2 of the human CD147 gene. The sgRNA sequence is ggagctgcactgcgaggccgtgg. Primers for amplifying 5' homologous arm: forward primer 5'-tggcgctgccaaaacttgtcaactgcgc-3', reverse primer 5'-cctctgctgggacagcggegcetgga-3'. Primers for amplifying 3' homologous arm: forward primer 5'-ctgggaggtgggtccagtctgag-3', reverse primer $5^{\prime}$-gcccaaaacatgccgagaggagtaaca-3'. A total of $1 \times 10^{6}$ cells were transfected by electroporation $(10 \mu \mathrm{g}$ plasmid $/ 1 \times 10^{6}$ cells $)$ and then plated on $100 \mathrm{~mm}$ culture dishes. 3 days after transfection, puromycin $(1 \mu \mathrm{g} / \mathrm{ml}$ for HCT- 8 and $3 \mu \mathrm{g} / \mathrm{ml}$ for HuH-7) was added to the culture medium, to allow single cell-derived colony formation. After puromycin selection, the survived clones were picked and expanded for genotyping.

\section{Quantitative real-time PCR analysis}

Quantitative real-time PCR analysis was performed as described previously [22]. Primer sequences were listed in Table 2.

\section{Nuclear/cytoplasmic fractionation}

Nuclear/cytoplasmic fractionation was performed using NE-PER ${ }^{\mathrm{TM}}$ Nuclear and Cytoplasmic Extraction Reagents (Pierce, US) according to the manufacturer's instructions. 


\section{In vitro wound-healing assay}

In vitro wound-healing assay was performed as described previously [29]. Briefly, $24 \mathrm{~h}$ after treatment, the cells were harvested and seeded in 12-well plates until confluent. A pipette was used to scratch the monolayer. The cells were then washed with serum-free medium. Photomicrographs were obtained at various time points ( $0 \mathrm{~h}$ and $24 \mathrm{~h}$ ), and the relative migration distance was calculated using the following formula: the relative migration distance $(\%)=100(\mathrm{AX}-\mathrm{BX}) /(\mathrm{A}$ blank - B blank), where $\mathrm{A}$ is the width of the cell wound before incubation, and B is the width of the cell wound after incubation.

\section{FRET assay}

The FRET assay was performed according to the methodology described previously [42-45]. Briefly, three sequential images were acquired with suitable filter sets for donor (EGFP; excitation at $488 \mathrm{~nm}$ and emission at 515 $\mathrm{nm}$ ), acceptor (DsRed; excitation at $543 \mathrm{~nm}$ and emission at $585 \mathrm{~nm}$ ), and FRET (excitation at $488 \mathrm{~nm}$ and emission at $585 \mathrm{~nm}$ ) under an oil immersion objective (n.a. $=1.40$ ). Donor and acceptor images were utilized to generate $\mathrm{CoA}$ and $\mathrm{CoB}$ data for the next FRET calculation with NISElements software (Nikon, Japan). The FRET efficiency calculation and image procession were performed with analysis software (Nikon, Japan) [46].

\section{Fluorometric analysis of the RhoA/Rac1 activation}

Cells were prepared as previously reported [47]. Images were obtained using an A1 confocal microscopy (Nikon, Japan). For emission ratio imaging, the filter sets were used as previously reported [48]. NIS-Elements software (Nikon, Japan) was used to analyze the images following previously described methods [47]. Briefly, images were dark-current and background-subtracted. Binary masks generated through intensity thresholding were applied to each emission channels, and the matched FRET and donor image sets were ratioed to depict Rac1 or RhoA activation throughout the cell. A linear pseudocolour lookup table was applied.

\section{In vitro kinase assay}

The his ${ }_{6}$-tagged proteins were purified as described previously [49]. Purified baculovirus-expressed human Src was obtained from Millipore. The kinase assay was performed as described previously [50]. Briefly, purified Annexin A2 and purified Src were incubated with or without purified CD147, C2 domain or I domain in kinase buffer (50mM HEPES $\mathrm{pH} 7.8,5 \mathrm{mM} \mathrm{MgCl}_{2}$, $150 \mathrm{mM} \mathrm{NaCl}, 1 \mathrm{mM}$ DTT, $1 \mathrm{mM}$ ATP) plus $1 \mathrm{mM}$ sodium orthovanadate at $30^{\circ} \mathrm{C}$ for 30 minutes. The reactions were stopped with the addition of Laemmli sample buffer before subjected to gel electrophoresis and Western blot.

\section{Surface plasmon resonance (SPR)}

SPR studies were performed using a BioRad ProteOn XPR36 instrument according to a One-shot Kinetics protocol $[51,52]$. GLC chips were initialized using glycerol and preconditioning was performed by sequential injections of $0.5 \% \mathrm{SDS}, 100 \mathrm{Mm} \mathrm{HCl}$, and then $50 \mathrm{mM} \mathrm{NaOH}$, each for $60 \mathrm{sec}$ at $25 \mu \mathrm{l} / \mathrm{min}$. The surface was then activated using $40 \mathrm{mM}$ EDAC and $10 \mathrm{mM}$ SulfoNHS, injected for $5 \mathrm{~min}$ at $25 \mu \mathrm{l} / \mathrm{min}$. $50 \mu \mathrm{g} / \mathrm{ml}$ of Annexin $\mathrm{A} 2$ in $10 \mathrm{mM}$ sodium acetate, $\mathrm{pH} 5.5$, was injected in the vertical direction over the desired channels for $6 \mathrm{~min}$ at $25 \mu \mathrm{l} / \mathrm{min}$. $50 \mu \mathrm{g} / \mathrm{ml}$ of BSA was also immobilized in the same way using as a control. The remaining activated carboxyl groups were blocked with a 3 min injection of $1 \mathrm{M}$ ethanolamine at $25 \mu \mathrm{l} / \mathrm{min}$ in the vertical direction. The chip was washed with PBS until a stable baseline was achieved. The running buffer was then switched to TBS with $0.05 \%$ Tween-20 (TBS-T). A concentration series of the analyte of interest was injected over the immobilized ligand (Annexin A2). Injection of the analyte diluted in TBS-T was done in the horizontal direction at $50 \mu \mathrm{l} / \mathrm{min}$ for $3 \mathrm{~min}$. An equivalent buffer injection was used for reference subtraction. The dissociation time was set to 12 $\mathrm{min}$. Equilibrium and rate constants were calculated using ProteOn Manager Software. A local $\mathrm{R}_{\max }$ value and the Langmuir model were used.

\section{Immunofluorescence}

Immunofluorescence was performed as described previously [22]. Briefly, cells were harvested and allowed to attach for $24 \mathrm{~h}$ to fibronectin-pre-coated cell culture dishes with glass bottoms (801002, NEST Biotechnology Co., LTD.). After washing twice with PBS, the cells were fixed in paraformaldehyde in PBS, permeabilized with $0.1 \%$ Triton $\mathrm{X}-100$, and blocked with $1 \%$ BSA in PBS for $1 \mathrm{~h}$. The dishes were first incubated with the indicated antibodies for $1 \mathrm{~h}$, washed twice with PBS, and then incubated with Alexa 488-phalloidin solution and the corresponding FITC-conjugated secondary antibodies for $30 \mathrm{~min}$ in the dark. Cell nuclei were dyed with DAPI (Vector Labs). After washing, the cells were visualized using an A1R-A1 confocal laser microscope system (Nikon, Japan). 


\section{Live-cell imaging}

For live-cell imaging [53], cells $\left(1 \times 10^{4}\right)$ were harvested at $24 \mathrm{~h}$ after transfection and allowed to attach for $12 \mathrm{~h}$ to fibronectin-pre-coated $35 \mathrm{~mm}$ dishes. Timelapse images using phase-contrast and fluorescence microscopy (A1R-A1 confocal laser microscope system, Nikon) were taken at 2 min intervals and assembled into movies using NIS-Elements software (Nikon, Japan). For quantitative image analysis of the lamellipodial extension after transfection of siDOCK3 or siCtrl, we took measurements of cell area changes by subtracting the areas of the cells at 0 min from those at 2 min using NISElements software (Nikon).

\section{In vivo metastasis assay}

Male BALB/c nude mice at 4 to 6 weeks of age were provided by the Laboratory Animal Research Center of FMMU, and the animal study was approved by the Animal Care and Use Committee of FMMU. The experimental metastatic potential of HCC cells was assessed following intrasplenic injection as described previously [40].

\section{ACKNOWLEDGMENTS}

We thank Louis Hodgson for sharing Rac1 biosensor, Gang Nan, Xiu-Xuan Sun, Yang Zhang and Yu-Kui Shang for technical help. This work was supported by the National Natural Science Foundation of China (31371405 and 81201774), the National High Technology Research and Development Program of China (2012AA020302) and the National S\&T Major Project (2012ZX10002017-002).

\section{CONFLICTS OF INTEREST}

The authors declare no conflict of interest.

\section{REFERENCES}

1. Yilmaz M and Christofori G. Mechanisms of motility in metastasizing cells. Molecular cancer research. 2010; 8:629-642.

2. Sadok A and Marshall CJ. Rho GTPases: masters of cell migration. Small GTPases. 2014; 5:e29710.

3. Yu XL, Hu T, Du JM, Ding JP, Yang XM, Zhang J, Yang B, Shen X, Zhang Z, Zhong WD, Wen N, Jiang H, Zhu P, et al. Crystal structure of HAb18G/CD147: implications for immunoglobulin superfamily homophilic adhesion. J Biol Chem. 2008; 283:18056-18065.

4. Zhao P, Zhang W, Tang J, Ma XK, Dai JY, Li Y, Jiang JL, Zhang SH and Chen ZN. Annexin II promotes invasion and migration of human hepatocellular carcinoma cells in vitro via its interaction with $\mathrm{HAb} 18 \mathrm{G} / \mathrm{CD} 147$. Cancer science. 2010; 101:387-395.

5. Zhao P, Zhang W, Wang SJ, Yu XL, Tang J, Huang W, Li Y, Cui HY, Guo YS, Tavernier J, Zhang SH, Jiang JL and Chen ZN. HAb18G/CD147 promotes cell motility by regulating annexin II-activated RhoA and Rac1 signaling pathways in hepatocellular carcinoma cells. Hepatology. 2011; 54:2012-2024.

6. Radke K and Martin GS. Transformation by Rous sarcoma virus: effects of src gene expression on the synthesis and phosphorylation of cellular polypeptides. Proceedings of the National Academy of Sciences of the United States of America. 1979; 76:5212-5216.

7. Glenney JR, Jr. Phosphorylation of $\mathrm{p} 36$ in vitro with pp60src. Regulation by $\mathrm{Ca} 2+$ and phospholipid. FEBS letters. 1985; 192:79-82.

8. He KL, Sui G, Xiong H, Broekman MJ, Huang B, Marcus AJ and Hajjar KA. Feedback regulation of endothelial cell surface plasmin generation by PKC-dependent phosphorylation of annexin A2. The Journal of biological chemistry. 2011; 286:15428-15439.

9. Brandherm I, Disse J, Zeuschner D and Gerke V. cAMPinduced secretion of endothelial von Willebrand factor is regulated by a phosphorylation/dephosphorylation switch in annexin A2. Blood. 2013; 122:1042-1051.

10. de Graauw M, Tijdens I, Smeets MB, Hensbergen PJ, Deelder AM and van de Water B. Annexin A2 phosphorylation mediates cell scattering and branching morphogenesis via cofilin Activation. Molecular and cellular biology. 2008; 28:1029-1040.

11. Kashiwa A, Yoshida H, Lee S, Paladino T, Liu Y, Chen Q, Dargusch R, Schubert D and Kimura H. Isolation and characterization of novel presenilin binding protein. Journal of neurochemistry. 2000; 75:109-116.

12. Chen Q, Peto CA, Shelton GD, Mizisin A, Sawchenko PE and Schubert D. Loss of modifier of cell adhesion reveals a pathway leading to axonal degeneration. The Journal of neuroscience. 2009; 29:118-130.

13. Tachi N, Hashimoto $\mathrm{Y}$ and Matsuoka M. MOCA is an integrator of the neuronal death signals that are activated by familial Alzheimer's disease-related mutants of amyloid beta precursor protein and presenilins. The Biochemical journal. 2012; 442:413-422.

14. Namekata K, Harada C, Taya C, Guo X, Kimura H, Parada LF and Harada T. Dock3 induces axonal outgrowth by stimulating membrane recruitment of the WAVE complex. Proceedings of the National Academy of Sciences of the United States of America. 2010; 107:7586-7591.

15. Namekata K, Harada C, Guo X, Kimura A, Kittaka D, Watanabe $\mathrm{H}$ and Harada T. Dock3 stimulates axonal outgrowth via GSK-3beta-mediated microtubule assembly. The Journal of neuroscience. 2012; 32:264-274.

16. Sanz-Moreno V, Gadea G, Ahn J, Paterson H, Marra P, Pinner S, Sahai E and Marshall CJ. Rac activation and 
inactivation control plasticity of tumor cell movement. Cell. 2008; 135:510-523.

17. Ladhani O, Sanchez-Martinez C, Orgaz JL, Jimenez B and Volpert OV. Pigment epithelium-derived factor blocks tumor extravasation by suppressing amoeboid morphology and mesenchymal proteolysis. Neoplasia. 2011; 13:633642.

18. Wright GJ. Signal initiation in biological systems: the properties and detection of transient extracellular protein interactions. Molecular bioSystems. 2009; 5:1405-1412.

19. Vetrivel KS, Zhang X, Meckler X, Cheng H, Lee S, Gong P, Lopes KO, Chen Y, Iwata N, Yin KJ, Lee JM, Parent AT, Saido TC, et al. Evidence that CD147 modulation of beta-amyloid (Abeta) levels is mediated by extracellular degradation of secreted Abeta. The Journal of biological chemistry. 2008; 283:19489-19498.

20. Dziduszko A and Ozbun MA. Annexin A2 and S100A10 regulate human papillomavirus type 16 entry and intracellular trafficking in human keratinocytes. Journal of virology. 2013; 87:7502-7515.

21. Hayes MJ and Moss SE. Annexin 2 has a dual role as regulator and effector of $\mathrm{v}$-Src in cell transformation. J Biol Chem. 2009; 284:10202-10210.

22. Wang SJ, Cui HY, Liu YM, Zhao P, Zhang Y, Fu ZG, Chen ZN and Jiang JL. CD147 promotes Src-dependent activation of Rac1 signaling through STAT3/DOCK8 during the motility of hepatocellular carcinoma cells. Oncotarget. 2015; 6:243-57. Doi: 10.18632/oncotarget.2801.

23. Rescher U, Ludwig C, Konietzko V, Kharitonenkov A and Gerke V. Tyrosine phosphorylation of annexin A2 regulates Rho-mediated actin rearrangement and cell adhesion. Journal of cell science. 2008; 121:2177-2185.

24. Babbin BA, Parkos CA, Mandell KJ, Winfree LM, Laur $\mathrm{O}$, Ivanov AI and Nusrat A. Annexin 2 regulates intestinal epithelial cell spreading and wound closure through Rhorelated signaling. The American journal of pathology. 2007; 170:951-966.

25. Garrido-Gomez T, Dominguez F, Quinonero A, Estella C, Vilella F, Pellicer A and Simon C. Annexin A2 is critical for embryo adhesiveness to the human endometrium by RhoA activation through F-actin regulation. FASEB J. 2012; 26:3715-3727.

26. Liu JW, Shen JJ, Tanzillo-Swarts A, Bhatia B, Maldonado CM, Person MD, Lau SS and Tang DG. Annexin II expression is reduced or lost in prostate cancer cells and its re-expression inhibits prostate cancer cell migration. Oncogene. 2003; 22:1475-1485.

27. Balch C and Dedman JR. Annexins II and V inhibit cell migration. Experimental cell research. 1997; 237:259-263.

28. Nakayama H, Fukuda S, Inoue H, Nishida-Fukuda H, Shirakata Y, Hashimoto K and Higashiyama S. Cell surface annexins regulate $\mathrm{ADAM}$-mediated ectodomain shedding of proamphiregulin. Molecular biology of the cell. 2012; 23:1964-1975.
29. Wang SJ, Cui HY, Liu YM, Zhao P, Zhang Y, Fu ZG, Chen ZN and Jiang JL. CD147 promotes Src-dependent activation of Rac1 signaling through STAT3/DOCK8 during the motility of hepatocellular carcinoma cells. Oncotarget. 2015; 6:243-257. Doi: 10.18632/oncotarget.2801.

30. Namekata K, Enokido Y, Iwasawa K and Kimura H. MOCA induces membrane spreading by activating Rac1. The Journal of biological chemistry. 2004; 279:1433114337.

31. Caspi E and Rosin-Arbesfeld R. A novel functional screen in human cells identifies MOCA as a negative regulator of Wnt signaling. Molecular biology of the cell. 2008; 19:4660-4674.

32. Takahashi K. WAVE2 Protein Complex Coupled to Membrane and Microtubules. Journal of oncology. 2012; 2012:590531.

33. Yamazaki D, Kurisu S and Takenawa T. Involvement of Rac and Rho signaling in cancer cell motility in $3 \mathrm{D}$ substrates. Oncogene. 2009; 28:1570-1583.

34. Xu HY, Qian AR, Shang P, Xu J, Kong LM, Bian HJ and Chen ZN. siRNA targeted against HAb18G/CD147 inhibits MMP-2 secretion, actin and FAK expression in hepatocellular carcinoma cell line via ERK1/2 pathway. Cancer letters. 2007; 247:336-344.

35. Jiang JL, Chan HC, Zhou Q, Yu MK, Yao XY, Lam SY, Zhu H, Ho LS, Leung KM and Chen ZN. HAb18G/CD147mediated calcium mobilization and hepatoma metastasis require both $\mathrm{C}$-terminal and $\mathrm{N}$-terminal domains. Cellular and molecular life sciences. 2004; 61:2083-2091.

36. Jiang JL, Zhou Q, Yu MK, Ho LS, Chen ZN and Chan HC. The involvement of HAb18G/CD147 in regulation of storeoperated calcium entry and metastasis of human hepatoma cells. J Biol Chem. 2001; 276:46870-46877.

37. Tang J, Wu YM, Zhao P, Yang XM, Jiang JL and Chen ZN. Overexpression of HAb18G/CD147 promotes invasion and metastasis via alpha3beta1 integrin mediated FAK-paxillin and FAK-PI3K-Ca2+ pathways. Cell Mol Life Sci. 2008; 65:2933-2942.

38. Dai JY, Dou KF, Wang CH, Zhao P, Lau WB, Tao L, $\mathrm{Wu}$ YM, Tang J, Jiang JL and Chen ZN. The interaction of HAb18G/CD147 with integrin alpha6betal and its implications for the invasion potential of human hepatoma cells. BMC Cancer. 2009; 9:337.

39. Li Y, Wu J, Song F, Tang J, Wang SJ, Yu XL, Chen ZN and Jiang JL. Extracellular membrane-proximal domain of HAb18G/CD147 binds to metal ion-dependent adhesion site (MIDAS) motif of integrin beta1 to modulate malignant properties of hepatoma cells. J Biol Chem. 2012; 287:47594772.

40. Wu J, Ru NY, Zhang Y, Li Y, Wei D, Ren Z, Huang XF, Chen ZN and Bian H. HAb18G/CD147 promotes epithelialmesenchymal transition through TGF-beta signaling and is transcriptionally regulated by Slug. Oncogene. 2011; 30:4410-4427. 
41. Li HW, Yang XM, Tang J, Wang SJ, Chen ZN and Jiang JL. Effects of HAb18G/CD147 knockout on hepatocellular carcinoma cells in vitro using a novel zinc-finger nucleasetargeted gene knockout approach. Cell biochemistry and biophysics. 2015; 71:881-890.

42. Almabouada F, Diaz-Ruiz A, Rabanal-Ruiz Y, Peinado JR, Vazquez-Martinez R and Malagon MM. Adiponectin receptors form homomers and heteromers exhibiting distinct ligand binding and intracellular signaling properties. J Biol Chem. 2013; 288:3112-3125.

43. Feige JN, Sage D, Wahli W, Desvergne B and Gelman L. PixFRET, an ImageJ plug-in for FRET calculation that can accommodate variations in spectral bleed-throughs. Microscopy research and technique. 2005; 68:51-58.

44. Gordon GW, Berry G, Liang XH, Levine B and Herman B. Quantitative fluorescence resonance energy transfer measurements using fluorescence microscopy. Biophysical journal. 1998; 74:2702-2713.

45. Xia Z and Liu Y. Reliable and global measurement of fluorescence resonance energy transfer using fluorescence microscopes. Biophysical journal. 2001; 81:2395-2402.

46. Liang Q, Han Q, Huang W, Nan G, Xu BQ, Jiang JL and Chen ZN. HAb18G/CD147 regulates vinculin-mediated focal adhesion and cytoskeleton organization in cultured human hepatocellular carcinoma cells. PloS one. 2014; 9:e102496.

47. Pertz O, Hodgson L, Klemke RL and Hahn KM. Spatiotemporal dynamics of RhoA activity in migrating cells. Nature. 2006; 440:1069-1072.

48. Fritz RD, Letzelter M, Reimann A, Martin K, Fusco L, Ritsma L, Ponsioen B, Fluri E, Schulte-Merker S, van Rheenen $\mathrm{J}$ and Pertz O. A versatile toolkit to produce sensitive FRET biosensors to visualize signaling in time and space. Science signaling. 2013; 6:rs12.

49. Zhang WJ, He YX, Yang Z, Yu J, Chen Y and Zhou CZ. Crystal structure of glutathione-dependent phospholipid peroxidase Hyr 1 from the yeast Saccharomyces cerevisiae. Proteins. 2008; 73:1058-1062.

50. Yu L, Thakur S, Leong-Quong RY, Suzuki K, Pang A, Bjorge JD, Riabowol K and Fujita DJ. Src regulates the activity of the ING1 tumor suppressor. PLoS One. 2013; 8:e60943.

51. Nahshol O, Bronner V, Notcovich A, Rubrecht L, Laune $\mathrm{D}$ and Bravman T. Parallel kinetic analysis and affinity determination of hundreds of monoclonal antibodies using the ProteOn XPR36. Anal Biochem. 2008; 383:52-60.

52. Bravman T, Bronner V, Lavie K, Notcovich A, Papalia GA and Myszka DG. Exploring "one-shot" kinetics and small molecule analysis using the ProteOn XPR36 array biosensor. Anal Biochem. 2006; 358:281-288.

53. Kato T, Kawai K, Egami Y, Kakehi Y and Araki N. Rac1dependent lamellipodial motility in prostate cancer PC-3 cells revealed by optogenetic control of Rac1 activity. PloS one. 2014; 9:e97749. 